\title{
3-dimensional arenas for the assessment of $C$. elegans behavior
}

\author{
Steel N. Cardoza ${ }^{1}$, Lai Yu Leo Tse², Kira Barton², Eleni Gourgou,2,3, *
}

${ }^{1}$ Materials Science and Engineering, University of Michigan, Ann Arbor, Ml

2 Mechanical Engineering, University of Michigan, Ann Arbor, MI

${ }^{3}$ Institute of Gerontology, Medical School, University of Michigan, Ann Arbor, MI

*: corresponding author (egourgou@umich.edu)

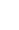

\section{Abstract}

C. elegans nematodes are a well-established model organism in numerous fields of experimental biology. In nature, $C$. elegans live in a rich 3-dimensional environment. However, their behavior has been assessed almost exclusively on the open, flat surface of NGM (Nematode Growth Medium) plates, the golden standard for $C$. elegans culture in the lab. We present two methods to build 3-dimensional behavioral arenas for $C$. elegans, by casting, and by directly 3D printing NGM hydrogel. The latter is achieved by using a highly customized fused deposition modeling (FDM) 3D-printer, modified to employ NGM hydrogel as ink. The result is the advancement of 3-dimensional complexity of behavioral assays. To demonstrate the potential of our method, we use the 3D-printed arenas to assess $C$. elegans physical barriers crossing. $C$. elegans decision to cross physical obstacles is affected by aging, physiological status (i.e., starvation), and prior experience. The 3D-printed structures can be used to spatially confine C. elegans behaviors, i.e., egg laying. We consider these findings a decisive step toward characterizing C. elegans 3-dimensional behavior, an area long overlooked due to technical constrains. We envision our method of 3D-printing NGM arenas as a powerful tool in behavioral neurogenetics, neuroethology, and invertebrate model organisms' neurobiology. 


\section{Keywords}

C. elegans, 3D-printing, 3-dimensional behavior, physical obstacles

\section{Introduction}

C. elegans nematodes are a well-established model organism in numerous fields of experimental biology, with prominent among them the biology of aging, behavioral neurogenetics, and neurobiology (1-4). In nature, C. elegans live in a rich 3-dimensional environment (e.g., rotten fruit, muddy soil) (5). However, their behavior has been assessed almost exclusively on the open, flat surface of NGM (Nematode Growth Medium) plates (6), which are the golden standard of $C$. elegans culture in the lab.

Recently, a new type of associative learning was reported (7), observed in Tshaped mazes (Fig. 1A). C. elegans learning to reach a target T-maze arm is related to the 3-dimensional nature of the arena $(7,8)$, namely walls, floor, and overall surfaces, which are perceived through multiple sensory modalities. In addition, $C$. elegans show a

44 clear preference for richly patterned surfaces (9). Combined, these findings support the idea that 3-dimensional environments are required to witness the full behavioral expression of these nematodes, and a larger part of their nervous system's capacity. $C$. elegans 3-dimensional locomotion is also gaining a lot of attention (10-13), revealing very interesting dynamics in both swimming and crawling nematodes.

To explore C. elegans spatial behavior, our group uses customized arenas (Fig.

1). To this end, 3D-printed plastic molds are used to imprint mazes of various designs in NGM undergoing solidification $(7,8)$. This leads to reliable, highly repeatable, and importantly, nematode-friendly assays because the generated arenas are built entirely out of NGM (Fig 1). However, several constrains apply. Although the complexity in the $x, y$ dimensions (plane parallel to the assay plate surface) can increase freely (Fig. 1A$D)$, complexity in $z$ dimension (plane perpendicular to the assay plate surface) is essentially limited to varying the depth of the arenas. Vertical elements, suspended

57 features, multi-level structures and other similar architectures are not allowed using molds.

To meet the need for alternative fabrication processes, we explored two 
61 synthetic polymer, to cast NGM structures (Figs 2 and 3). The ensuing parts are of high

62 quality and provide valuable feedback regarding the NGM self-sustaining properties.

63 However, this method's limitations motivated us to seek another route, one that employs

64 a 3D printer, which uses NGM as ink.

65 A growing number of researchers are employing 3Dprinting as a transformative

66 tool for cell and tissue engineering $(14,15)$. This includes 3-dimensional scaffolds made

67 of enriched hydrogel-based materials (16). Hydrogels of $1 \%-5 \%$ agar concentrations

68 have been successfully explored for 3D bioprinting applications (17). Most of the

69 occurring structures are sturdily self-supported cubes or other non-hollow, no-overhang

70 designs. Interestingly, the 3D-printing technology has not been used to produce

71 behavioral arenas for the study of small invertebrate animal models, like C. elegans.

We present a highly customized prototype 3D-printer, the Parnon Printer

73 (Parnon: mountain in southern Greece, known for its many gorges), which can print 3-

74 dimensional parts, suitable for C. elegans behavioral experiments, made of $2 \%$ agar-

75 based NGM hydrogel. The resulting arenas are nematode-friendly, minimizing the

76 stress that could have been induced when the animals are transferred from the culture

77 plate into the arenas.

78 To demonstrate the suitability of the Parnon-printed parts, we use them to assess

79 C. elegans physical barrier crossing ability, in the context of aging (young, middle-aged

80 adults), feeding history (fully fed, starved animals) and prior experience (have been or

81 not in the presence of a 3D structure before). We also explore the usage of 3D-printed

82 structures to spatially confine C. elegans egg laying behavior. C. elegans behavior in 3-

83 dimensional environments is by definition not possible to explore on standard flat NGM

84 plates. Therefore, the findings reported here would likely not have been brought to light

85 if the Parnon Printer had not been developed.

\section{Results}

$88 \quad$ PVA casted 3D-structures

89 The first fabrication method explored was PVA casting (Fig. 2). The produced structures

90 show that it is possible to create 3-dimensional NGM structures in a systematic and

91 predictable way. 
Specifically, the crossbridge design showed that NGM can support itself, even at a height of $4 \mathrm{~mm}$ and an overhang length of $12 \mathrm{~mm}$, with legs $2 \times 2 \mathrm{~mm}$ in cross section(Fig 2B). Self-standing crossbridges are also feasible in smaller structures (Fig. 2D), $2 \mathrm{~mm}$ high and $5 \mathrm{~mm}$ long overhang, where the bridge legs are thinner, $1.5 \mathrm{~mm}$ in cross section. This means that the mechanical properties of NGM $2 \%$ in agar are such that allow for overhangs which stretch a few worm body lengths long, without the need of extra support. It is noted that during the solidification process, NGM enjoyed the support provided by the PVA cast itself (Fig. 2A).

Cross sectional dimensions of beams to as small as $1 \times 1 \mathrm{~mm}$ have been attempted but were found too small to consistently allow NGM to enter all the way into the cast channels (Fig 2D, missing right arm). This was similarly the case when casting the diving bell design (Fig. 2C, 3B), where the cross section of the arms was also $1 \times 1 \mathrm{~mm}$.

PVA casting produces parts with very rough surfaces (Fig. 3). This is a consequence of the cast 3D-printing process, during which the PVA is laid in a way that allows micropockets of air among the deposed PVA threads. These micropockets get filled later with NGM, thus creating tiny protrusions (Fig. 3C, green arrows).

\section{Parnon customization and Parnon-printed 3D-structures}

We extensively modified the commercially available FDM (Fusion Deposition Modeling) printer of choice (Fig. 4, Supplementary Fig. S1) and converted it in a highly customized hydrogel-ink 3D printer, named Parnon. Customization included wideranging modifications of the print head and substrate (Fig. 4C, 4D, Supplementary Figs. S2, S3A), and involved design, 3D-printing (motor arm housing, syringe plunger connector), and machining (print head aluminum heat sink) of tailored parts (Supplementary Fig. S1). Effective synergy of all parts was imperative for the successful operation of the instrument.

Parnon printer can successfully use NGM as ink to print 3-dimensional structures

120 (Fig. 5 and Suppl. Fig. 7). Although the examples presented here are of lower

121 complexity compared to PLA and other plastic or resin 3D-printed objects, essential

122 properties of 3D-printed structures are achieved. Hence, the Parnon-printed parts 
123 consist of multiple layers (up to 3), making this is an effective way to increase vertical

124 complexity of behavioral arenas, in the dimension perpendicular to the surface of NGM

125 plates. More layers are mechanically feasible, however currently Parnon does not allow

126 for very precise stacking of deposed layers, which results in the top layers and bottom

127 layers being misaligned (Fig. 5B).

128 Importantly, the Parnon-printed parts have smooth surfaces, especially when

129 they are printed using a narrower nozzle and higher resolution printing head (Fig. 5A,

$1305 \mathrm{~B}, 5 \mathrm{E}, 5 \mathrm{~F})$. The extrusion is unhindered and continuous during a $90^{\circ}$ angle turn

131 (example: Fig. 5E, framed area) and NGM lines can be laid successfully in $90^{\circ}$ angles or

132 smaller (Suppl. Fig. 7B).

C. elegans ability to cross physical barriers, and the effect of aging

To confirm that Parnon-printed structures can be used to investigate $C$. elegans behavior, we ran a series of experiments. In the first scheme, we assessed nematodes' ability to cross physical barriers, in order to reach a food source (Figs 6 and 7). We also examined the role of aging (Fig. 6), feeding history, and prior experience (Fig. 7).

To establish that the 3D printed squares constitute a physical barrier for $C$. elegans, we conducted a control experiment, in which adult Day 1 nematodes are allowed to reach a food source not framed by a physical barrier (Fig. 6B, and 6D, left). Animals reached the food containing area in large numbers quickly, and almost all of them (96-100\%) remained there for most of the 120min assay (Fig. 6C, grey circles).

Next, we challenged adult Day $1 \mathrm{C}$. elegans with a food source, framed by a 20x20mm 3D-printed NGM square (Fig. 6D, right). In this case, nematodes entered the framed area gradually and in lower rates, and at the end of the $120 \mathrm{~min}$ assay, $74 \%$ of animals had crossed the square barrier and had reached the food source (Fig. 6C, black circles). Therefore, the NGM square frame presents a physical barrier for nematodes.

We hypothesized that decision making related to physical challenges differs in young and old animals, as aging-driven behavioral changes have been broadly reported in $C$. elegans (18-20). To test our hypothesis, we ran the square barrier experiment with 153 adult Day 7 nematodes. In contrast to young adults, only $41 \%$ of Day 7 C. elegans have 
154

155

crossed the barrier after 120min (Fig. 6C, purple diamonds). When adult Day 7 nematodes were tested without a physical barrier (control experiment), results were similar to the ones of adult Day 1 nematodes (Fig. 6C, pink diamonds). Since Day 7 adults are beyond middle age, the above findings suggest an aging-related change in $C$. elegans physical barrier crossing behavior.

\section{The effect of confined area size}

Next, we asked whether the size of the confined area and its ratio over the total available plate area affects the dynamics of the assay, i.e., whether worms travel in and out of the target area or stay inside the target square once they cross it.

To this end, we ran the same experiment, using a $5 \times 5 \mathrm{~mm}$ 3D-printed NGM square instead of a $20 \times 20 \mathrm{~mm}$ one. Indeed, when the $20 \times 20 \mathrm{~mm}$ square was used (Fig. 6), the nematodes that crossed the barrier stayed inside the framed area for the remainder of the assay. In case of the $5 \times 5 \mathrm{~mm}$ square, (Fig. $7 \mathrm{C}$ vs Fig. $6 \mathrm{~B}$ ), nematodes did not remain inside the square once they enter, instead they might enter, roam, exit, and even reenter. Due to this dynamic situation, the number of worms counted inside the square did not increase monotonically, as in Fig. 6, instead, it fluctuated. For this reason, and to reflect this dynamic behavior, instead of a time course (Fig. $6 \mathrm{C}$ ), results are presented as scatter plots (Fig. 7A, 7B), showing the mean and standard deviation of the percentage of worms scored inside the framed area at any given time point.

In addition, the $20 \times 20 \mathrm{~mm}$ square (Fig. 6) frames $\sim 15 \%$ of the $60 \mathrm{~mm}$ plate surface area, whereas the $5 \times 5 \mathrm{~mm}$ one (Fig. 7) frames $<1 \%$ of that area. This is probably why a smaller percentage of worms is scored inside the $5 \times 5 \mathrm{~mm}$ square at any given moment, compared to what happens with the $20 \times 20 \mathrm{~mm}$ one. Therefore, the relative size of the square with respect to the culture plate affects the dynamics of the assay and should be taken into consideration when data is interpreted.

\section{The effect of feeding history}

When two groups of Day 1 adults were tested (Fig. 7A), a fully fed one (FF; purple inverted triangles) and one that was starved for $24 \mathrm{hrs}$ prior testing (S; blue diamonds) it was found that starved animals enter the food-baited square in higher 
numbers than fully fed ones. This suggests that starved animals might have a stronger motivation to explore their surrounding area and possibly to overcome physical obstacles, as well.

When the experiment was performed with no OP50 inside the square (Fig. 7A, woF-without food, shaded area, vs wF-with food, non-shaded area) C. elegans behaved similarly, and starved animals (S woF, blue circles) entered the food-baited square in higher numbers than fully fed ones (FF woF, purple triangles). Therefore, the nematodes' feeding history has a strong influence on their tendency to explore beyond a physical barrier even if there is no food beyond it.

\section{The effect of prior experience}

Lab populations of $C$. elegans are commonly grown either in liquid cultures or on flat NGM plates with practically 2-dimensional surfaces. C. elegans used in the present work have been cultured for many generations on NGM plates. Hence, we asked whether a group of nematodes that has dwelled on a NGM plate featuring a 3D-printed square will have familiarized themselves with it, and thus will cross a similar 3D barrier in higher numbers. To this end, we placed L4 nematodes on a seeded NGM plate with a 5X5mm Parnon-printed square, for 24 hours. Then, and on Day 1 of their adult life, we tested them on a different plate, equipped with a similar, food-baited $5 \times 5 \mathrm{~mm}$ square.

Nematodes that have previously experienced an environment which includes a 3D square (Fig. 7B, 3D), cross the barrier in higher rates than the ones that have experienced only the flat surface of a regular NGM plate $(R)$. This is the case regardless of whether the 3D structure frames a food baited area (wF) or a non-baited area (woF, shaded).

\section{Spatial restriction of egg laying}

We explored whether the Parnon-printed squares can be used to spatially control selected $C$. elegans behaviors, i.e., egg laying. To this end, a 5x5mm Parnon-printed square, baited with OP50, was used. (Fig. 8). We used the small size squares to apply a stricter constrain on the behavior we aim to control. Results showed that Day 1 adults that were placed on the plate and were left there for 24 hours, laid eggs almost 
exclusively in the confined area or on the square barriers themselves, since almost no

217 eggs are found at other plate locations (Fig. 8A-D).

\section{Discussion}

Less than a handful of attempts has been made to date to establish wormfriendly and experimentally informative 3-dimensional arenas for C. elegans (21-23). In two of these studies, the 3-dimensional platforms are made of porous materials,

223 primarily intended for cultivating and imaging worms $(21,23)$. In the third case $(22)$,

224 nematodes swim in microfluidic devices that resemble their granulated natural

225 environment, i.e. soil. These substrates provide a significantly more realistic terrain than

226 the NGM plate, however each of them lacks one of the key traits (well-defined and

227 structured arenas, compatibility with microscopy and imaging techniques, or easy and

228 cheap fabrication) that would allow it to be widely adopted for 3-dimensional behavioral 229 experiments. The methods presented here, especially the Parnon NGM printing, aspire 230 to fill this gap.

PVA-casted 3dimensional structures

PVA casting successfully results in creating diverse 3-dimensional NGM

234 structures (Fig. 2). However, there are important downsides. When the chambers of the

235 PVA cast (Fig. 2A) are small ( 1mm in diameter), NGM is not casted properly. This could be related to the fact that small spaces result in faster cooling of NGM, and subsequent clogging. A possible way to resolve this would be to keep the cast warm throughout the process to prevent premature NGM solidification.

At the same time, some of the cast channels might be partially blocked, due to 240 trapped air bubbles or 3D printing discrepancies, occasionally happening when such 241 small empty spaces need to be achieved. A 3D printer with higher resolution than the

242 Ultimaker3 could potentially help resolve this issue, increasing, however, the overall 243 cost of production.

$244 \quad$ Lastly, PVA casting results in parts with very rough surfaces (Fig. 3). The 245 increased roughness of the NGM surfaces may or may not be a desired feature, 246 depending on the experiment to be conducted. Indeed, strong roughness could possibly 
247 impede or redirect nematode locomotion or interfere with optics and imaging. Note, for

248 example, how challenging is to distinguish a nematode that is crawling on the NGM

249 casted structures of Fig 3 (3A: yellow arrow, 3B, 3C: yellow boxes).

250 In addition, PVA casting process is time consuming, as it takes 28 hours per

251 part to complete (see Methods). It is also resource heavy, since PVA is significantly

252 more expensive than most PLA or other standard plastic filaments. Moreover, the casts

253 are not reusable, so a new cast must be printed and dissolved for each new NGM part.

254 Furthermore, access to a high-resolution printer, like the Ultimaker3, is required. In

255 addition, certain features cannot be obtained using casts, as for example complex

256 vertical elements, sharp angles, and very small size features. Consequently, PVA casts

257 do not constitute a go-to option. This outcome prompted us to explore the 3D-printing

258 route discussed below.

Parnon customization and Parnon-printed 3D-structures

The printability of hydrogels has been broadly demonstrated (15, 24-26), including the extrusion of agarose-based hydrogels $(27,28)$. The $2 \%$ agar concentration in the NGM mix is among the lowest used in hydrogel 3D printing $(17,26,29)$ without

264 the use of enhancers (30). Moreover, the Parnon-produced structures presented here

265 and used in C. elegans behavioral experiments feature three layers of NGM. This

266 constitutes a promising achievement when compared to previously reported lattices and

267 scaffolds, made with hydrogel ink of similar concentration and properties (15), mainly

268 because it was possible without additional support (31). In coarse trials where multi-

269 layer cylinders or walls were attempted, although the stability of the structures was 270 satisfying, the overall printing quality was low and inconsistent (Supplementary Fig. S9).

$271 \quad$ NGM lines can be laid successfully in $90^{\circ}$ angles or smaller (Suppl. Fig. 7B). This

272 allows for a diverse set of designs, although the printing quality is compromised

273 (Supplementary Fig. S7B, yellow frame). Printing lines in sharp angles is in general a 274 challenging task (26) and the performance of Parnon is currently considered satisfactory

275 for the current experimental needs. Stricter control of the extrusion process would

276 improve this aspect (26). 
In general, the resolution of the FDM-printed structures is restricted by the nozzle diameter (32), which is limited by clogging, and depends on the rheological properties of the extruded material. Hence, the typical resolution for FDM is $\sim 100 \mu \mathrm{m}$ (32). When it comes to bioprinting hydrogel inks that contain biomolecules, the typical size of the printed features is $\sim 500 \mu \mathrm{m}$ (32). According to other reports (33), the achieved resolution in non-hydrogel specific FDM printing is around 50/250 $\mu \mathrm{m}(z / x y)$, while older actuation pressure extrusion attempts using hydrogels (34) report a $z / x y$ resolution of $500 \mu \mathrm{m}$.

284 The resolution achieved by Parnon reaches $300 \mu \mathrm{m}$ in $z$, and $\sim 250 \mu \mathrm{m}$ in $x y$. Therefore, Parnon's performs well regarding its $x y$ resolution, when compared to overall FDM printing, and scores better than other hydrogel-ink printers. Parnon's $z$ resolution ranks close to its hydrogel-ink peers but is considerably worse than generally achieved FDM z resolution. Better control of the actuation pressure and extruded NGM viscosity could help improve this property. Given the size of adult $C$. elegans' body, i.e. $\sim 1 \mathrm{~mm}$ length and 70-100 $\mu$ m diameter (35), a moderate upgrade would probably suffice for most applications. Nevertheless, the resolution achieved by the current version of Parnon serves well the experimental purpose of the printed parts.

Compared to the PVA-casted parts, Parnon-printed structures are smoother (Fig. 5 vs Fig. 3), allowing for easier imaging of nematodes (Fig. 5B inset). Apart from the initial purchasing cost of the commercial printer, 3D-printing hydrogel arenas using Parnon is an affordable and fast way to create 3-dimensional assays, especially when compared to the PVA casting.

Parnon mechanics, extrusion, and software communication

Currently, pausing and resuming printing is a challenging process. Unlike market FDM 3D printers, the extrusion of which can start and stop easily on demand, Parnon still struggles to do that quickly and precisely. The result is undesired dripping of NGM, even when the actuation pauses or reverses. Because of this, the printing process needs to be continuous (Supplementary Fig. 7), thus affecting the printing paths of 305 certain designs (e.g., cross shape, Supplementary Fig. 7C). Deeper understanding of 306 the compressive behavior and solidification kinetics of NGM, more efficient control of 
307 the nozzle heating, or interventions at the extrusion pathway could result in more

308 precise extrusion control and ultimately allow for more tailored designs.

Parnon's custom print head is not compatible with the basis printer firmware and

thus requires its own commands. Market available gCode slicers do not have the capability to output relevant and processable linear actuator commands in the Parnon's format. The development of the gCode and Arduino commands remains a rigorous manual process. We are working toward implementing appropriate gCode slicers in

314 future iterations of the prototype.

To demonstrate the suitability of the Parnon-printed parts as C. elegans behavioral arenas, we showed that three-layered NGM squares can be successfully used to explore nematodes' ability to cross physical barriers (Fig. 6). This is the first attempt to explore $C$. elegans ability to overcome physical obstacles, made of the same nematode-friendly material on which worms are cultured in the lab.

The height of the square used (Fig. 6), is estimated at $\sim 1.5 \mathrm{~mm}$ (three layers, $0.5 \mathrm{~mm}$ thick each), which equals approximately 1.5 times the adult $C$. elegans' body length. This means that the hurdle is not extraordinarily high, compared to C. elegans, yet it is hard to establish a reference, since similar experiments have not been conducted before. Having in mind the 3-dimensional character of $C$. elegans' natural habitats $(5,36)$, we speculate that an environment with $1.5 \mathrm{~mm}$ high features is not out of range for a field-dwelling nematode. Therefore, we assume that such encounters are not uncommon for $C$. elegans in the field. Hence, the finding that young adult worms can easily climb over them is not surprising. It is noted that in addition to nematodes which climb over the square barrier, $\sim 5 \%$ of the population tested crawled between the square and the substrate on which it rested.

Basic motor skills and chemotaxis remain functional through later stages of adulthood in C. elegans nematodes (37), however some decline in locomotion speed (38) and spontaneous locomotion has been reported $(19,39)$. The observed difference 336 between Day 1 and Day 7 adults (Fig. 6) could be attributed to age-affected locomotion 337 performance. 
Results from dispersal assays have shown that young adult $C$. elegans move away from the original position and spread into a wide area on the assay plate, but older adults are more likely to remain close to their original location (19). This agrees with our findings on Day 7 adult nematodes, which do not spread over the square barrier as their Day 1 conspecifics (Fig. 6). Dispersal over physical obstacles has not been previously assessed. Notably, a non-negligible part of the Day 7 population in study did cross the barrier, revealing a diversity in individual $C$. elegans behavior (40) and maybe a diverse impact of aging-related changes (41).

It is reported that learning and memory decline earlier in adulthood than other physiological operations $(7,37,42)$ and show signs of deterioration on Day 7 (37) or even Day 5 of $C$. elegans adult life (7). It is possible that some aspects of decision making, e.g., related to overcoming physical challenges, are affected, as well. Moreover, a decrease in food-seeking exploration in older nematodes is possible. starvation-induced modifications of nematode behavior that improve their food location likelihood. Indeed, after prolonged starvation, C. elegans change their strategy to longrange dispersal (43-46). Even after 10-20 min away from food, neuronal plasticity can change, leading to global, instead of local, search behavior in adult nematodes (47). Moreover, it has been shown that food deprivation increases threat tolerance in $C$. elegans, resulting in worms that make "bolder" decisions (48). Broadening the explored area, even if it includes some physical challenges, may be an additional manifestation of behavioral changes triggered by food deprivation. Feeding history seems to impact obstacle crossing more than the presence or absence of food inside the framed area (Fig. 7B). generations on NGM plates, conforming with common practice in the field.

364 Consequently, the 3D-printed squares are practically the first 3-dimensional structure they encounter, besides the unfriendly walls of the plastic petri dish itself and the NGM chunk often used in culture maintenance, when transferring nematodes into a fresh culture plate. In case of the latter, $C$. elegans usually actively interact with the chunk, but they commonly just crawl away from it, since the chunk is flipped over the fresh 
369 NGM plate (6). Our findings (Fig. 7B) suggest that C. elegans behavior with regards to

370 physical challenges is affected by their prior experience and familiarity with similar

371 structures. We expect that this effect will be stronger if the animals are grown in a 3-

372 dimensional environment since hatching, and more 3-dimensional structures exist in

373 their culture plate.

Spatial confinement of C. elegans behavior

Given that in the above experiments (Fig. 7) C. elegans get in and out of the square frames without much difficulty, the localized egg laying behavior (Fig. 8) can be attributed to the fact that the only food source available is located inside the 3D squares. This makes them a preferable location for egg laying since this way the newly hatched progeny has immediate access to food. Indeed, it is known that egg laying is regulated by a number of environmental conditions (49), leading to spatial control of the egg laying behavior. Moreover, the egg laying rate in the presence of abundant food is significantly higher than in the absence of food (50). The use of the 3D squares works well with localized food availability and it proves very effective when it comes to restricting egg laying, and potentially the hatching of L1 larvae, as well.

Conclusions

This is the first reported attempt to explore $C$. elegans ability to cross physical obstacles, made of the same nematode-friendly material on which worms are cultured in the lab. A method that enables fabrication of such structures, as is the prototype NGMdemonstrate that new tools and assays are not only the key to answering biological questions but, most importantly, can often shape the very questions posed. We envision the applications of 3D printing NGM to enable artificial landscaping and development of

\section{Methods}

In this section we describe the PVA casting and the Parnon printing methods, 
400 customization, NGM rheological properties, and software communication between the

401 printer's parts can be found in the Supplementary Information section. A list of the major

402 components used for the conversion of the commercial printer into Parnon us provided

403 in the Appendix. Details on the Arduino code are provided in the Appendix.

404 The material used to build 3D-behavioral arenas is NGM (Nematode Growth

405 Medium), the agar-based hydrogel used to culture C. elegans in the lab. NGM $2 \%$ in

406 agar was prepared according to standard methods $(6,51)$. Once all the ingredients are

407 diluted in water, NGM is in liquid form in temperatures higher than $\sim 45^{\circ} \mathrm{C}$ (melting point),

408 and it solidifies as it cools down. In room temperature, polymerized NGM is solid. This is

409 a key property for both the PVA casting and Parnon 3D printing methods.

PVA casting-PolyVinylAlcohol; PVOH; $[\mathrm{CH} 2 \mathrm{CH}(\mathrm{OH})] n$ )

This approach takes advantage of the water solubility properties of PVA. To

413 design the cast, SolidWorks (Dassault Systemes, France) was used. The cast was

414 printed at an Ultimaker3 3D printer (Ultimaker BV, United States). The casts shown in

415 Fig. 2 were printed in $\sim 5$ hours. Once the cast was ready, liquid NGM was injected in.

416 NGM took the shape of the space that is left vacant inside the cast. Once the NGM

417 solidified thoroughly, after $\sim 30 \mathrm{~min}$, the cast was immersed in deionized water and was

418 sonicated for $\sim 24 \mathrm{hr}$ in a sonicator bath (Branson Ultrasonics, USA). This accelerated

419 dissolving of the PVA cast, which was entirely dissolved and was therefore not

420 reusable. Since NGM is not water soluble after polymerization, at the end of this

421 process only the NGM structure was left intact.

Fused Deposition Modeling (FDM) 3D printer frame

We used a commercially available FDM printer, the DOBOT MOOZ-2 machine

425 (Shenzhen, China, Fig. 4). It was preferred against other options due to i) existing

426 gCode interpretation firmware that controls the linear actuators in the cartesian axis

427 system, ii) price efficiency, as a result of low build volume, and iii) frame rigidity.

428 According to the manufacturer, MOOZ-2 is made of aircraft grade aluminum alloy, which

429 minimizes in situ vibrations and increases rigor. 
All three axes are controlled with lead screw linear actuators which are more accurate, precise, repeatable and user-friendly than the alternative. The MOOZ-2 is amenable to modifications (Fig. 4, Supplementary Fig. S1) that allow Parnon's custom print head and substrate to be attached.

\section{Parnon custom substrate}

$436 \quad$ Parnon's substrate is designed to limit liquid spreading and promote build up

437 along the z-axis (Fig. 4). The custom substrate consists of three major parts: i) the 438 plotting medium (Fig. 4 Part Di \& ii), ii) the cooling apparatus (Fig. 4 Part Diii \& iv), and 439 iii) the leveling system (Fig. 4 Part Dv).

We use a plotting medium $(34,52,53)$ to reduce liquid spreading, provide

443 glycerin (Sigma Aldrich, USA) in water. It has the same density as liquid NGM (1.024

$444 \mathrm{~g} / \mathrm{mL}$ ), so the extruded NGM is effectively suspended during the print to assist in limiting 445 liquid spreading. A 60mm diameter glass petri dish (Fig. 4 Part Di), holds $20 \mathrm{~mL}$ of the 446 plotting medium for each printing session.

447 ii. Cooling apparatus

NGM is heated by the heating element when inside the printing head (Fig. 4C,

449 Supplementary Figure S3A). Moreover, there is Joule heating around the nozzle, to 450 keep the NGM liquid during extrusion (Supplementary Figure S3). To cool down the 451 plotting medium and facilitate NGM solidification, a Peltier device (Northbear

452 Electronics) is used (26) (Fig. 4 Part Diii), operating in 16V 12A. This device cools the 453 plotting medium according to the thermoelectric effect (Fig. 4 Part D, red arrows). An 454 aluminum heat sink (Fig. 4 Part Div) is implemented underneath the Peltier device to 455 dissipate heat and ultimately increase its efficiency. The Parnon's cooling mechanism 456 operates at $86 \%$ efficiency, achieving a $2.0^{\circ} \mathrm{C}$ temperature decrease from the surface of 457 the plotting medium to the floor after 20min (Supplementary Fig. S6). Details on the 458 efficiency of the Peltier device and the heat flux generated are provided in the 459 Supplementary Information. 
The Parnon uses four individually adjustable spring resistance screws (Fig. 4 Part Div, Dv) to allow for substrate leveling. Additional circular levels with adjustable screws are placed on the petri dish (Fig. 4 Part Di, Supplementary Fig. S2).

\section{Parnon custom print head}

The custom print head consists of three major parts: i) a mechanism to house and heat NGM (Fig. 2 Part Civ, v, vii, viii, ix \& x), ii) gCode and Arduino software communication, and iii) a mechanism to provide actuation pressure (Fig. 2 Part Ci \& iii).

The aluminum heat sink (Fig. 2 Part Cv) was machined from 6020 aluminum. It connects all the components on the custom print head to Parnon's $x$ axis. The heat sink

472 is heated with a 3" 3/8" diameter 200W 120V heating element with an internal k-type

473 thermocouple (Fig. 4 Part Civ), which is controlled by a programmable temperature

474 controller. The temperature controller is set at $65^{\circ} \mathrm{C}$ because we expect $\mathrm{G}^{\prime}=\sim 5 \mathrm{~Pa}$

475 (Supplementary Fig. S6). This modulus is sufficiently low to allow easy extrusion and 476 sufficiently high to limit liquid spreading. Copper wire with 5V potential (Fig 4. Part Cx,

477 Supplementary Fig. S3) heats the nozzle (joule heating effect) so NGM does not solidify 478 while in the nozzle.

479 The current version of Parnon uses a $3 \mathrm{~mL}$ glass syringe (Fig. 4 Part Cviii,

480 Supplementary Fig. S3) to house liquid NGM for extrusion. An early version used a $5 \mathrm{~mL}$ 481 glass syringe. The glass plunger (Fig. 4 Part Cvii) is connected to the linear actuator 482 arm (Fig 4. Part Ciii) via a custom 3D-printed connector (Fig. 4 Part Cvi, Supplementary

483 Fig. S3, Formlabs Tough Resin), designed using Solidworks (Dessault Systemes, 484 France).

$485 \quad$ NGM is extruded through a $1 / 2$ " stainless-steel luer-lock nozzle. Various gauges of 486 luer-lock nozzles were tested (higher gauge translates in lower inner diameter). The 487 initial nozzle had a $813 \mu \mathrm{m}$ ID, we improved to a $404 \mu \mathrm{m}$ ID nozzle, and the current 488 version features a $254 \mu \mathrm{m}$ ID nozzle. Print resolution improves as the ID of the nozzle 489 decreases (Fig. 5, Supplementary Fig. S7).

490 ii. gCode and software communication 
Modifying the hardware of an existing 3D printer compromised the communication the printer had with the original print head. To resolve this issue, we introduced a limit switch, an Arduino card, and a stepper motor driver. More details are provided in the Supplementary Information section.

iii. Actuation pressure

A linear actuator facilitates extrusion by compressing NGM in the syringe. A characteristic stress is required for the NGM to begin flowing through the nozzle. The time required to reach stress $\left(t_{\text {eq. }}\right.$ ) is the target of our analysis of NGM under actuation pressure. Time $t_{e q}$. is used to guide the amount of time prior to print extrusion beginning dedicated to reaching the characteristic stress (strain). More details are provided in the Supplementary Information section.

NGM material characterization

NGM $2 \%$ agar was prepared according to standard methods $(6,51)$. The density of liquid NGM was experimentally evaluated to be $\sim 1.024 \mathrm{~g} / \mathrm{mL}$ ( $\sim 10 \mathrm{~mL}$ of NGM weight $\sim 10.24 \mathrm{~g}$, and the density of deionized water was considered $1.0 \mathrm{~g} / \mathrm{mL}$ ). The melting point of agarose (molecular biology grade, Sigma Aldrich, USA) is $\leq 65^{\circ} \mathrm{C}$ and the transition temperature (gel point) is $36^{\circ} \mathrm{C} \pm 1.5^{\circ} \mathrm{C}$ (for $1.5 \%$ gel), according to the manufacturer. 100microliters of blue food color (Americolor, CA, USA) were added to $100 \mathrm{~mL}$ of $\mathrm{NGM}(0.1 \%)$ for in situ visibility and observational purposes.

Rheology experiments were performed on NGM to uncover the fastest solidification temperature, using a TH DR2 Rheometer (TA Instruments, USA). The

514 temperature was dropped from $60^{\circ} \mathrm{C}$ to $25^{\circ} \mathrm{C}$ at a rate of $5^{\circ} \mathrm{C} / \mathrm{min}$. The optimal 515 solidification temperature is determined by the maximum slope of the G' vs T curve

516 (Supplementary Fig. S6). The slope (dG'/dT) peaks at $35.9^{\circ} \mathrm{C}$ (Supplementary Fig. S6),

517 indicating the fastest solidification temperature.

518 ii. NGM compressive viscoelastic response

$519 \quad$ NGM presents a viscoelastic response to compressive stress. Compressive 
521 glass syringe. Time $t_{e q}$ is required to reach the inflection point of the extrusion

522 equilibrium stress $\left(\sigma_{e q}\right.$. $)$ under actuation pressure, and time $t_{r}$ is required to relax from

523 it. The inflection point occurs at a stress value $\sigma_{e q}$. which varies with strain rate $\dot{\varepsilon}$. The

524 time $t_{e q}$. required to reach it, varies depending on syringe ID.

525 Details on NGM viscoelastic response characterization are provided in the

526 Supplementary Information section.

C. elegans behavioral experiments

529 Snapshots of $C$. elegans' actions during behavioral experiments were taken with 530 a DP22 camera, mounted on a SZ61 dissection microscope, using CellSens Software

531 (all by Olympus, Japan).

532 i. Barrier crossing

533 In the control experiment, where C. elegans are allowed to reach a food source 534 not framed by a physical barrier, a droplet of OP50 was placed on a regular NGM plate, 535 and was framed by a figurative square, drawn with a marker on the bottom of the plate. 536 Nematodes were placed around the targeted area (Fig. 6D, left) and were allowed to 537 roam free.

538 In all experiments where Parnon-printed squares were used (Figs 6,7 and 8),

539 the 3D structures were rinsed multiple times with deionized water and then were placed 540 on a $60 \mathrm{~mm}$ NGM plate. Next, when applicable, a of E. coli OP50 was pipetted gradually 541 inside the square and was left to dry for $\sim 10 \mathrm{~min}$ (10microliters for Fig. 6 experiments, 5

542 microliters for Figs. 7 and 8 experiments). A number of $C$. elegans nematodes were 543 then transferred on the NGM plate, outside of the square(s), at least 3mm away, at 544 random locations.

545 To explore the effect of aging on crossing physical barriers (Fig. 6), two age 546 cohorts of adult hermaphrodites were tested, namely young adults of Day 1 (L4+1), and 547 middle-aged animals of Day 7 (L4+7). We used a $\sim 20 \times 20 \mathrm{~mm} 3 \mathrm{D}$-printed square, made 548 of 3 NGM layers, $0.5 \mathrm{~mm}$ thick each (see Methods). We considered to the time point at 549 which all nematodes were placed on the plate. The worms were allowed 120min to 550 explore, and worms scored inside the square were counted every 5 or $10 \mathrm{~min}$.

551 Nematodes that were on the square were counted towards successful crossings. The 
552 E. coli OP50 food used in all trials came from the same stock batch, and experiments 553 were run on 3 different days.

$554 \quad$ To explore the effect of feeding history (Fig 7A, 7B) we tested young adults of

555 Day $1(L 4+1)$, and middle-aged animals of Day 7 (L4+7), that had either been fully fed

556 (FF) or starved for $24 \mathrm{hrs}(\mathrm{S})$. To explore the effect of prior experience (Fig. 7C) we

557 tested young adults of Day $1(\mathrm{~L} 4+1)$ that had been either grown on a regular NGM plate

$558(\mathrm{R})$ or had been moved into an NGM plate containing a Parnon-printed square, similar

559 to the one used in testing (3D). In all cases (Fig. 7A, 7B, 7C) both a baited (wF) and a

560 non-baited 3D square (woF) were tested. Moreover, in all cases (Fig. 7A, 7B, 7C) we

561 used $\sim 5 \times 5 \mathrm{~mm}$ squares, consisting of 3 layers $0.5 \mathrm{~mm}$ thick each.

562 ii. Spatial control of egg laying behavior

For these experiments (Fig. 8), 5x5mm3D-printed NGM squares consisting of 3

564 layers $0.5 \mathrm{~mm}$ thick each, were used. After printing, the squares were rinsed with

565 deionized water, and then were placed on a $60 \mathrm{~mm}$ NGM plate. Next, 5 microlitters of $E$.

566 coli OP50 were gradually pipetted inside the square area and were left to dry for

$567 \sim 10 \mathrm{~min}$. A population of Day $1(\mathrm{~L} 4+1)$ adult C. elegans hermaphrodites was then

568 transferred on the NGM plate and were placed at least 3mm away from the squares.

569 The plate was checked for eggs after $24 \mathrm{hrs}$.

C. elegans strains

$572 \quad$ N2 Bristol (wild type) C. elegans were used in all experiments, initially acquired

573 from CGC (Caenorhabditis Genetics Center, provided by the C. elegans Reverse

574 Genetics Core Facility at the University of British Columbia, which is part of the

575 international C. elegans Gene Knockout Consortium) and maintained in the lab.

Statistical analyses

$578 \quad$ Statistical evaluations were made using t-tests (GraphPad Prism 9.0.0) with $p$ values considered significant when $p>0.05$. In Figure 6, multiple unpaired t-tests were

580 performed, and in Figure 7 evaluations were made using two-tailed, unpaired t-tests.

581 Additional information is provided in the figures' captions. 


\section{Acknowledgements}

584 We thank Ao-Lin Hsu for the use of space and equipment, and Nikos Chronis for the use 585 of space. We are grateful to Zijun (Justin) Yuan for hydrogel testing, to Chris Pannier for 586 technical advice, to Bill Kirkpatrick and Kent Pruss for assistance with machining, to Alex 587 Shorter for use of Ultimaker3 3Dprinter, to Michael Solomon and Yufei Wei for rheometry 588 advice, to Arthur Sinclair for rheometer facilitation, and to Ruiming Lu for help with glass cutting. This work was funded by the University of Michigan Office of Research (UMOR)590 Faculty Grants \& Awards Program (EG). EG is the recipient of a NIH-NIA K01 award. The 591 content is solely the responsibility of the authors and does not necessarily represent the 592 official views of the National Institutes of Health.

\section{Authors' Contributions}

595 SNC designed and printed the PVA molds, built the Parnon printer, performed analyses, 596 collected data, generated figures, and wrote the manuscript. LYLT assisted with 597 customizing the commercial printer, and reviewed manuscript. KB contributed advice 598 and expertise, and reviewed manuscript. EG conceived the idea, designed Parnon 599 experiments, designed and performed C. elegans experiments, analyzed data, 600 generated figures, wrote, and edited the manuscript, and supervised research.

\section{References}

603 1. Tissenbaum HA. Using C. elegans for aging research. Invertebrate Reproduction \& 604 Development. 2015;59(sup1):59-63.

605 2. Corsi AK, Wightman B, Chalfie M. A Transparent Window into Biology: A Primer on Caenorhabditis elegans. Genetics. 2015;200(2):387-407.

3. Kaletta T, Hengartner MO. Finding function in novel targets: C. elegans as a model organism. Nature Reviews Drug Discovery. 2006;5(5):387-99. 4. Rankin $\mathrm{CH}$, Beck CD, Chiba CM. Caenorhabditis elegans: a new model system for the study of learning and memory. Behav Brain Res. 1990;37(1):89-92. 6. Stiernagle T. Maintenance of C. elegans, in WormBook ed. 2006. 7. Gourgou E, Adiga K, Goettemoeller A, Chen C, Hsu A-L. Caenorhabditis elegans

615 8. Gourgou E, Hsu A-L. A maze platform for the assessment of Caenorhabditis elegans 616 behavior and learning. STAR Protocols. 2021:100829.

617 9. Han B, Dong Y, Zhang L, Liu Y, Rabinowitch I, Bai J. Dopamine signaling tunes spatial 618 pattern selectivity in C. elegans. eLife. 2017;6:e22896. 
619

620

621

622

623

624

625

626

627

628

629

630

631

632

633

634

635

636

637

638

639

640

641

642

643

644

645

646

647

648

649

650

651

652

653

654

655

656

657

658

659

660

661

662

663

664

665

666

667

668

10. Bilbao A, Patel AK, Rahman M, Vanapalli SA, Blawzdziewicz J. Roll maneuvers are essential for active reorientation of Caenorhabditis elegans in 3D media. Proceedings of the National Academy of Sciences. 2018.

11. Shaw M, Zhan H, Elmi M, Pawar V, Essmann C, Srinivasan MA. Three-dimensional behavioural phenotyping of freely moving $\mathrm{C}$. elegans using quantitative light field microscopy. PLOS ONE. 2018;13(7):e0200108.

12. Kwon N, Hwang AB, You Y-J, V. Lee S-J, Ho Je J. Dissection of C. elegans behavioral genetics in 3-D environments. Scientific Reports. 2015;5:9564.

13. Kwon N, Pyo J, Lee S-J, Je JH. 3-D worm tracker for freely moving C. elegans. PloS one. 2013;8(2):e57484-e.

14. Li H, Tan C, Li L. Review of 3D printable hydrogels and constructs. Materials \& Design. 2018;159:20-38.

15. Li J, Wu C, Chu PK, Gelinsky M. 3D printing of hydrogels: Rational design strategies and emerging biomedical applications. Materials Science and Engineering: R: Reports. 2020;140:100543.

16. Spicer CD. Hydrogel scaffolds for tissue engineering: the importance of polymer choice. Polymer Chemistry. 2020;11(2):184-219.

17. Unagolla JM, Jayasuriya AC. Hydrogel-based 3D bioprinting: A comprehensive review on cell-laden hydrogels, bioink formulations, and future perspectives. Appl Mater Today. 2020;18:100479.

18. Stein GM, Murphy CT. The Intersection of Aging, Longevity Pathways, and Learning and Memory in C. elegans. Front Genet. 2012;3:259.

19. Glenn CF, Chow DK, David L, Cooke CA, Gami MS, Iser WB, et al. Behavioral Deficits During Early Stages of Aging in Caenorhabditis elegans Result From Locomotory Deficits Possibly Linked to Muscle Frailty. The Journals of Gerontology Series A: Biological Sciences and Medical Sciences. 2004;59(12):1251-60.

20. Golden TR, Hubbard A, Dando C, Herren MA, Melov S. Age-related behaviors have distinct transcriptional profiles in Caenorhabditis elegans. Aging cell. 2008;7(6):850-65.

21. Tahernia M, Mohammadifar M, Choi S. Paper-Supported High-Throughput 3D Culturing, Trapping, and Monitoring of Caenorhabditis Elegans. 2020;11(1).

22. Lockery SR, Lawton KJ, Doll JC, Faumont S, Coulthard SM, Thiele TR, et al. Artificial

Dirt: Microfluidic Substrates for Nematode Neurobiology and Behavior. Journal of Neurophysiology. 2008;99(6):3136-43.

23. Lee TY, Yoon K-H, Lee Jl. Cultivation of Caenorhabditis elegans in Three Dimensions in the Laboratory. Journal of visualized experiments : JoVE. 2016(118):55048.

24. Tavana H, Mosadegh B, Takayama S. Polymeric aqueous biphasic systems for noncontact cell printing on cells: engineering heterocellular embryonic stem cell niches. Advanced materials (Deerfield Beach, Fla). 2010;22(24):2628-31.

25. Chung JHY, Naficy S, Yue Z, Kapsa R, Quigley A, Moulton SE, et al. Bio-ink properties and printability for extrusion printing living cells. Biomaterials Science. 2013;1(7):763-73.

26. He Y, Yang F, Zhao H, Gao Q, Xia B, Fu J. Research on the printability of hydrogels in 3D bioprinting. Scientific reports. 2016;6:29977-.

27. Yan Y, Wang X, Xiong Z, Liu H, Liu F, Lin F, et al. Direct Construction of a Threedimensional Structure with Cells and Hydrogel. Journal of Bioactive and Compatible Polymers. 2005;20(3):259-69.

28. Ozbolat IT, Hospodiuk M. Current advances and future perspectives in extrusion-based bioprinting. Biomaterials. 2016;76:321-43.

29. Kessel B, Lee M, Bonato A, Tinguely Y, Tosoratti E, Zenobi-Wong M. 3D Bioprinting of Macroporous Materials Based on Entangled Hydrogel Microstrands. Advanced Science. 2020;7(18):2001419. 
669

670

671

672

673

674

675

676

677

678

679

680

681

682

683

684

685

686

687

688

689

690

691

692

693

694

695

696

697

698

699

700

701

702

703

704

705

706

707

708

709

710

711

712

713

714

715

716

717

718

30. Stanton MM, Samitier J, Sánchez S. Bioprinting of 3D hydrogels. Lab on a Chip. 2015;15(15):3111-5.

31. Fan R, Piou M, Darling E, Cormier D, Sun J, Wan J. Bio-printing cell-laden Matrigelagarose constructs. Journal of Biomaterials Applications. 2016;31(5):684-92.

32. Fan D, Staufer U, Accardo A. Engineered 3D Polymer and Hydrogel Microenvironments for Cell Culture Applications. Bioengineering (Basel). 2019;6(4):113.

33. Gross BC, Erkal JL, Lockwood SY, Chen C, Spence DM. Evaluation of 3D Printing and Its Potential Impact on Biotechnology and the Chemical Sciences. Analytical Chemistry. 2014;86(7):3240-53.

34. Landers R, Hübner U, Schmelzeisen R, Mülhaupt R. Rapid prototyping of scaffolds derived from thermoreversible hydrogels and tailored for applications in tissue engineering. Biomaterials. 2002;23(23):4437-47.

35. White JG, Southgate E, Thomson JN, Brenner S. The Structure of the Nervous System of the Nematode Caenorhabditis elegans. Philosophical Transactions of the Royal Society of London B, Biological Sciences. 1986;314(1165):1-340.

36. Schulenburg H, Félix M-A. The Natural Biotic Environment of Caenorhabditis elegans. Genetics. 2017;206(1):55-86.

37. Kauffman AL, Ashraf JM, Corces-Zimmerman MR, Landis JN, Murphy CT. Insulin Signaling and Dietary Restriction Differentially Influence the Decline of Learning and Memory with Age. PLoS Biol. 2010;8(5):e1000372.

38. Hsu A-L, Feng Z, Hsieh M-Y, Xu XZS. Identification by machine vision of the rate of motor activity decline as a lifespan predictor in C. elegans. Neurobiology of aging. 2009;30(9):1498-503.

39. Newell Stamper BL, Cypser JR, Kechris K, Kitzenberg DA, Tedesco PM, Johnson TE. Movement decline across lifespan of Caenorhabditis elegans mutants in the insulin/insulin-like signaling pathway. Aging cell. 2018;17(1):e12704.

40. Stern S, Kirst C, Bargmann Cl. Neuromodulatory Control of Long-Term Behavioral Patterns and Individuality across Development. Cell. 2017;171(7):1649-62.e10.

41. Ahadi S, Zhou W, Schüssler-Fiorenza Rose SM, Sailani MR, Contrepois K, Avina M, et al. Personal aging markers and ageotypes revealed by deep longitudinal profiling. Nature Medicine. 2020;26(1):83-90.

42. Schreiber MA, Pierce-Shimomura JT, Chan S, Parry D, McIntire SL. Manipulation of Behavioral Decline in Caenorhabditis elegans with the Rag GTPase raga-1. PLoS genetics. 2010;6(5):e1000972.

43. Hills T, Brockie PJ, Maricq AV. Dopamine and glutamate control area-restricted search behavior in Caenorhabditis elegans. J Neurosci. 2004;24(5):1217-25.

44. Tsalik EL, Hobert O. Functional mapping of neurons that control locomotory behavior in Caenorhabditis elegans. Journal of Neurobiology. 2003;56(2):178-97.

45. Wakabayashi T, Kitagawa I, Shingai R. Neurons regulating the duration of forward locomotion in Caenorhabditis elegans. Neuroscience research. 2004;50(1):103-11.

46. Gray JM, Hill JJ, Bargmann Cl. A circuit for navigation in Caenorhabditis elegans.

Proceedings of the National Academy of Sciences of the United States of America. 2005;102(9):3184-91.

47. López-Cruz A, Sordillo A, Pokala N, Liu Q, McGrath PT, Bargmann Cl. Parallel Multimodal Circuits Control an Innate Foraging Behavior. Neuron. 2019;102(2):407-19.e8.

48. Ghosh DD, Sanders, T., Hong, S., McCurdy, L. Y., Chase, D. L., Cohen, N., Koelle, M. R., Nitabach, M. N. Neural Architecture of Hunger-Dependent Multisensory Decision Making in C. elegans. Neuron. 2016;92(5):1049-62.

49. Schafer WR. Egg-laying. 2005. In: WormBook : the online review of $\mathrm{C}$ elegans biology [Internet]. 
719 50. Trent C, editor Genetic and behavioral studies of the egg-laying system in

720 Caenorhabditis elegans1983.

721

722

723

724

51. Brenner S. The genetics of Caenorhabditis elegans. Genetics. 1974;77(1):71-94.

52. Wei J, Wang J, Su S, Wang S, Qiu J, Zhang Z, et al. 3D printing of an extremely tough hydrogel. RSC Advances. 2015;5(99):81324-9.

53. Hinton TJ, Jallerat Q, Palchesko RN, Park JH, Grodzicki MS, Shue HJ, et al. Threedimensional printing of complex biological structures by freeform reversible embedding of

727

728

729

730

731

732

733

734

735

736

737

738

739

740

741

742

743

744

745

746

747

748

749

750 

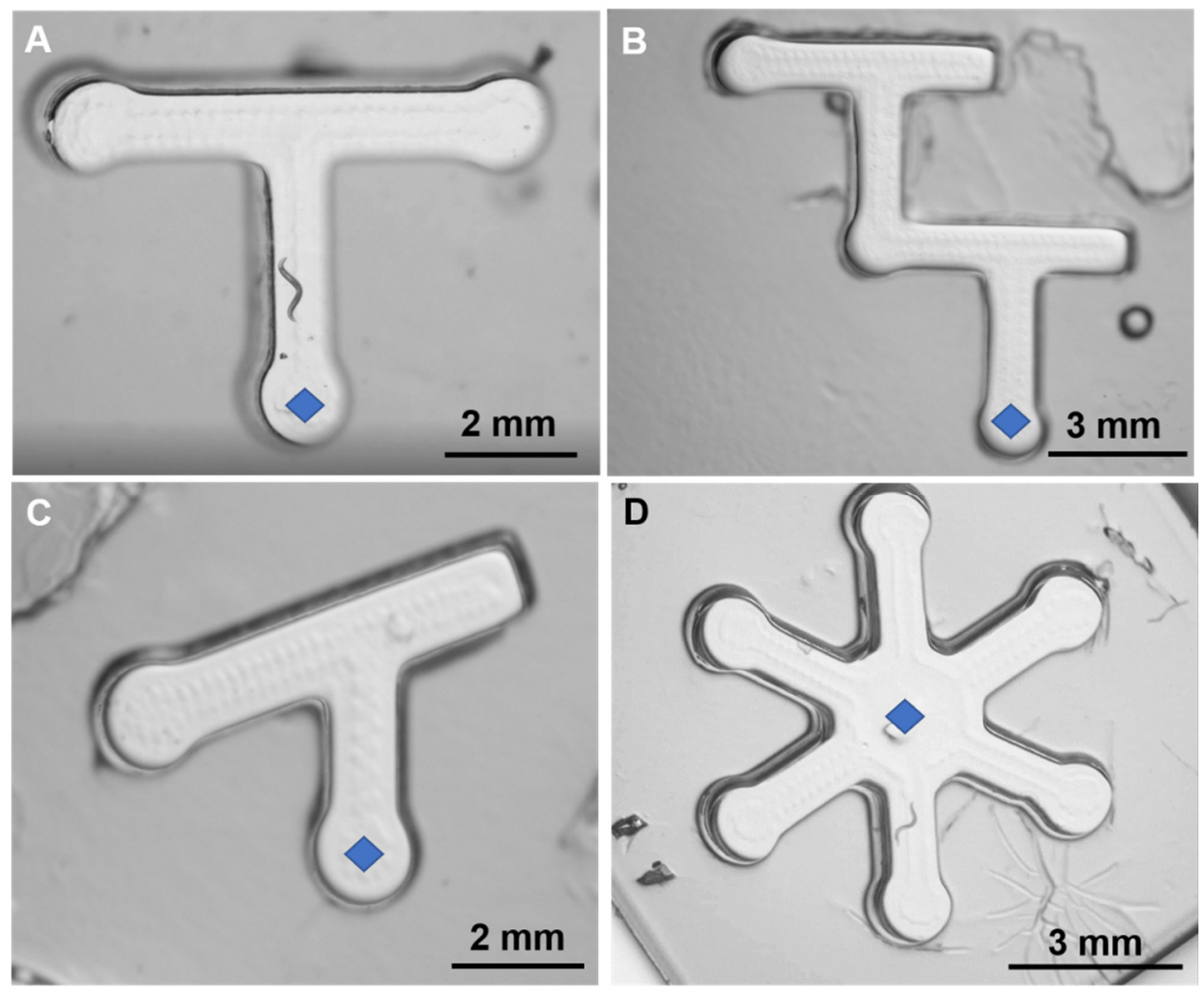

756 Figure 1: NGM behavioral arenas for C. elegans, made with molding technique. (A) A simple, symmetrical T-maze (see also Gourgou et al, 2020). (B) Double T-maze, a more complex version of T-maze. (C) Angled T-maze with asymmetrical ends. (D)

759 Radial arena, with six arms. All panels: mazes/arenas depth: $\sim 5 \mathrm{~mm}$; diamond shape 760 indicates the intended assay starting point. 

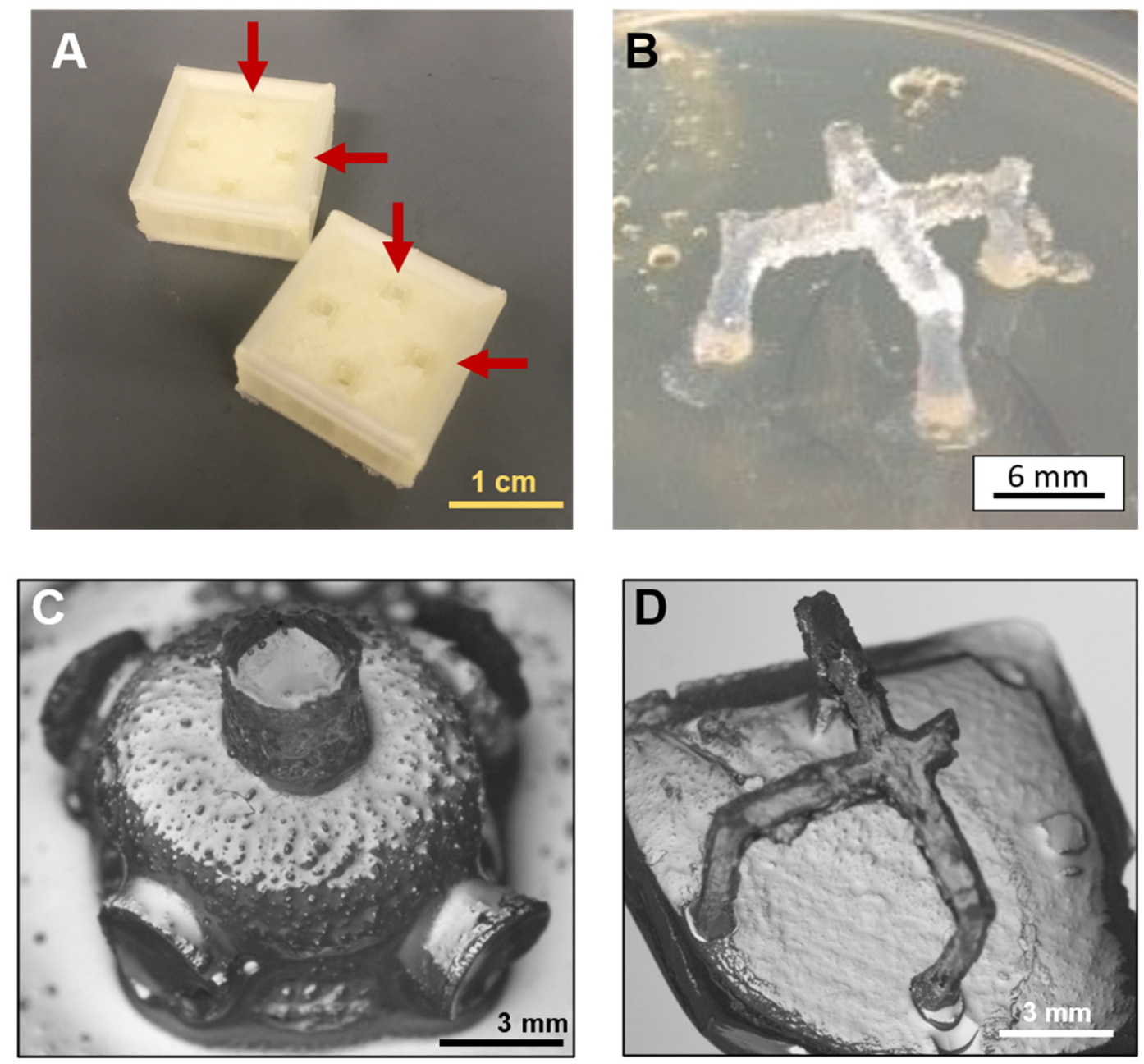

767 Figure 2: NGM 3-dimensional structures, made with the PVA casting method. (A)

768 Two 3D-printed PVA casting molds, red arrows indicate the liquid NGM input point. (B) 769 A four-legged NGM crossbridge, made using one of the casts shown in $(A)$, sitting on a

770 NGM plate surface. Legs are slightly tilted outwards because of flipping and handling

771 the structure. (C) A diving bell - like structure, consisting of a hemisphere (radius: $5 \mathrm{~mm}$ )

772 and designed to have five cylindrical arms, diameter: $3 \mathrm{~mm}$, length: $1-1.5 \mathrm{~mm}$, each.

773 Liquid NGM did not reach the entire length of the hollow space inside the PVA cast,

774 resulting in much shorter arms than originally planned $(4 \mathrm{~mm})$. Note the rough surface

775 of the structure. (D) A four-legged crossbridge standing on a $2 \mathrm{~mm}$ thick base, raised 4

$776 \mathrm{~mm}$ above the base's surface. This is a much thinner structure than the one in (B),

777 showcasing the self-supporting properties of NGM even in smaller arrangements. Note 778 the missing right arm. 

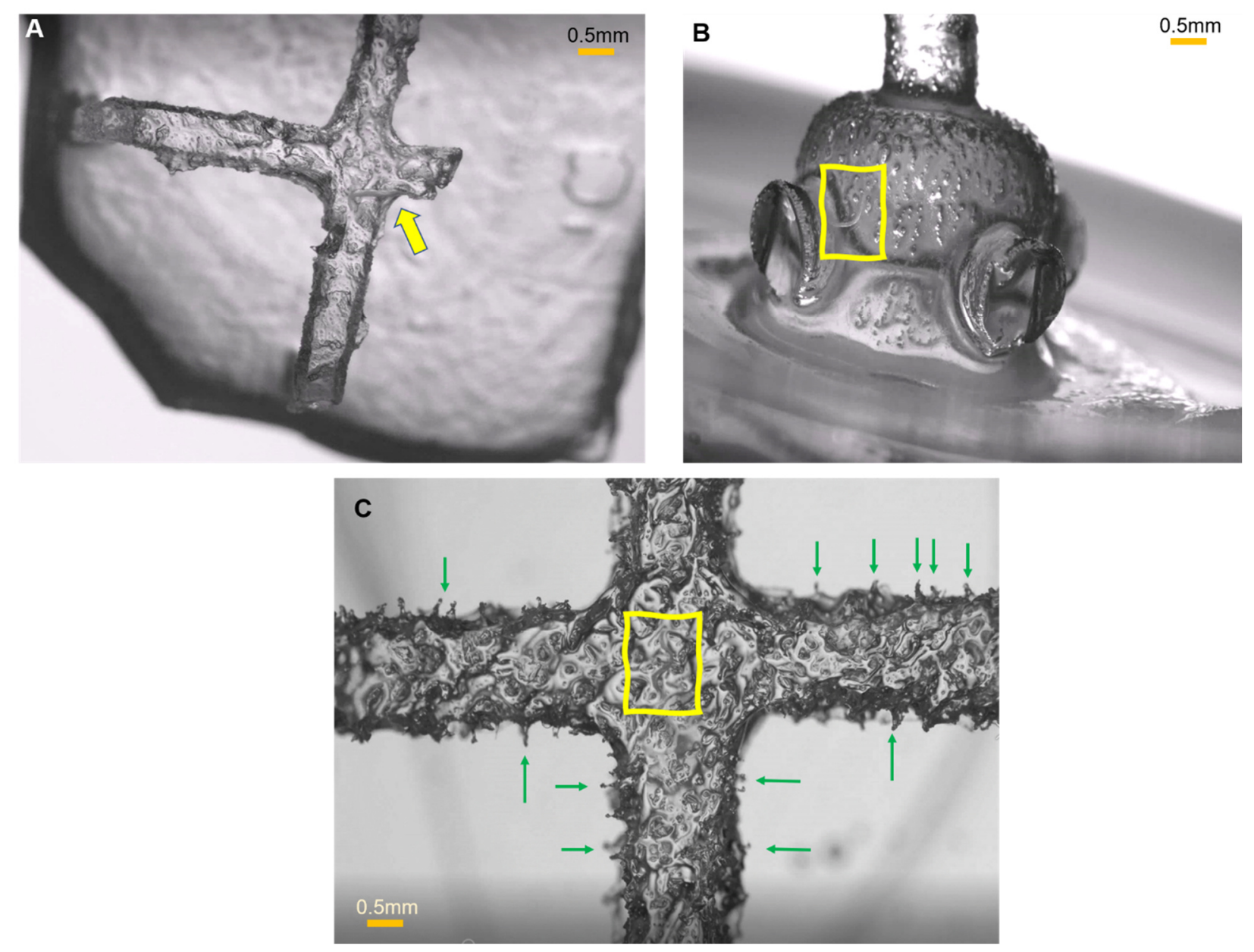

Figure 3: Properties of PVA-casted 3-dimensional NGM structures. (A) Closeup of the four-legged NGM crossbridge, shown in Fig. 2D, top view. Yellow arrow points to a C. elegans nematode crawling on the upper surface, at the corner between two beams. Note the rough surface and the thin, relatively to worm's body length, structure. (B) Closeup of the NGM diving bell-like structure, shown in Fig. 2C, side view. Yellow frame indicates the position of a $C$. elegans nematode. Note the bumpy surface and the incomplete beams. (C) Closeup of the four-legged NGM crossbridge, shown in Fig. 2B,

788 top view. Yellow frame indicates the position of a C. elegans nematode. Note the very 789 rough surface, of which the protruding features (examples highlighted with green 790 arrows) are similar to or even bigger than the worm's body width. The C. elegans worm 791 is challenging to distinguish in all three photos. 

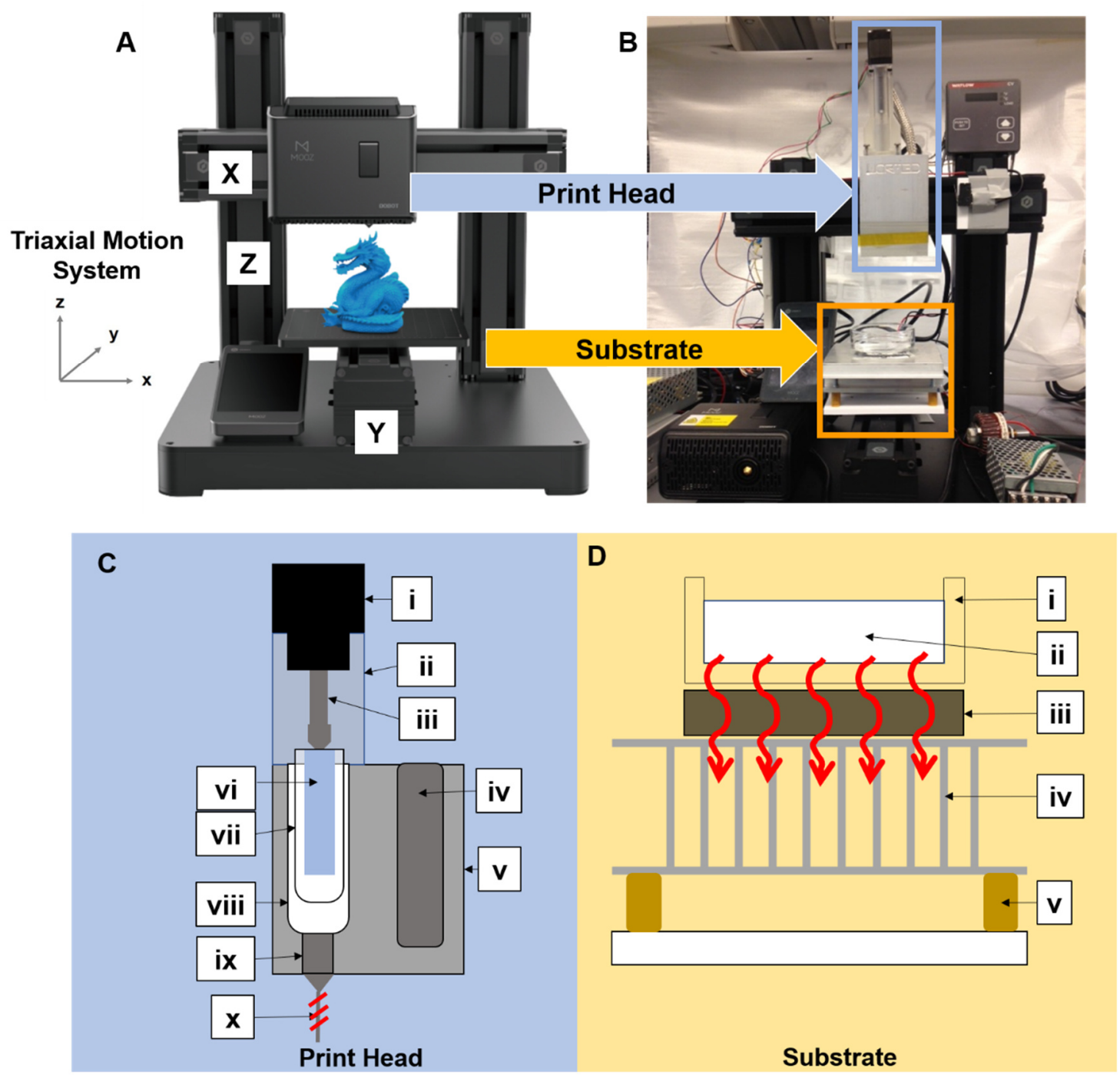

Figure 4: Overview of customization changes that convert a FDM printer to Parnon Printer. (A) The FDM 3D Printer DOBOT MOOZ (image courtesy of the manufacturer). The three main parts are the print head, the substrate, and the tri-axial relative motion system. While the stock motion system was considered adequate, both the print head and substrate had to be modified. (B) The prototype Parnon Printer,

800 featuring a customized print head and substrate, designed for printing NGM. See also 801 Supplementary Fig. S1. (C) Customized print head, front view schematic, connected to 802 the $x$ axis of the triaxial motion system. i) Bipolar stepper motor of the linear actuator. ii) 803 3D-printed custom connector, houses the stroke arm (iii) and connects the motor (i) to 804 the heat sink (v). iii) Stroke arm of the linear actuator. iv) Heating element, heats the 
805 aluminum sink, keeping NGM in liquid state. v) Custom aluminum heat sink. vi) Custom

806 connector connects the stroke arm (iii) to the plunger (vii). vii) Glass syringe plunger

807 adhered on the custom connector. viii) Glass syringe houses the liquid NGM. ix) Metal

808 luer-lock nozzle. $x$ ) Copper wire heat induction system, to prevent NGM solidification in

809 the nozzle (ix). Details on the print head parts in Appendix. (D) Customized substrate,

810 front view schematic, connected to the $Y$ axis of the triaxial motion system. i) Glass petri

811 dish. ii) Plotting medium. iii) Peltier device, red arrows indicate direction of heat transfer.

812 iv) Heat sink to promote the thermal gradient created by the Peltier device (iii). iv)

813 Springs used as a four-point bed leveling system (Supplementary Fig. S2). 

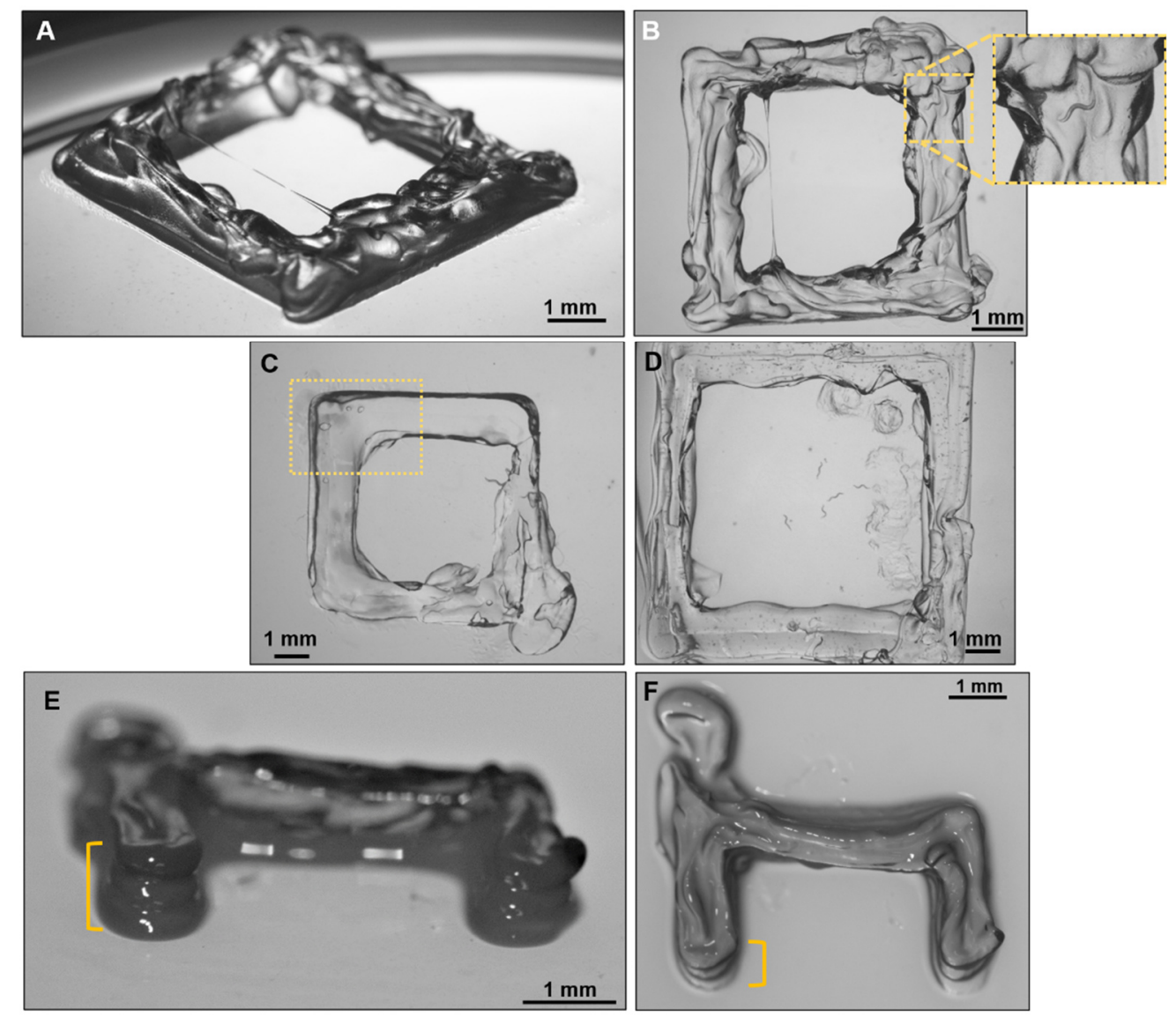

Figure 5: Parnon-printed NGM 3-dimensional structures. The Parnon-printed 3D

structures have visibly smoother surface compared to cast-made ones (see Fig. 3). (A)

835 Perspective and (B) top view of a $7 \times 7 \mathrm{~mm}$ square-shaped print, consisting of 3 layers,

$836500 \mu \mathrm{m}$ thick each. Printing conditions: head speed $10 \mathrm{~mm} / \mathrm{s}$, actuation speed $31 \mu \mathrm{m} / \mathrm{s}$,

837 printing head specs: nozzle ID $813 \mu \mathrm{m}$, linear actuator NEMA 14. Inset: a clearly

838 distinguishable nematode, crawling on the printed NGM. (C) Top view of a 7x7mm

839 square print, consisting of 3 layers, $300 \mu \mathrm{m}$ thick each. Printing conditions: head speed

$84010 \mathrm{~mm} / \mathrm{s}$, actuation speed $38 \mu \mathrm{m} / \mathrm{s}$, printing head specs: nozzle ID $254 \mu \mathrm{m}$, linear

841 actuator NEMA 8. Yellow frame indicates the smoothly deposed NGM line at the corner

842 of the square $\left(90^{\circ}\right.$ angle deposition). (D) Top view of a $20 \times 20 \mathrm{~mm}$ square print,

843 consisting of 3 layers, $300 \mu \mathrm{m}$ thick each. Printing conditions: head speed $10 \mathrm{~mm} / \mathrm{s}$,

844 actuation speed $8 \mu \mathrm{m} / \mathrm{s}$, printing head specs: nozzle ID $254 \mu \mathrm{m}$, linear actuator NEMA 8.

845 (E) Perspective and (F) top view of a C-shaped print, consisting of 3 layers, $300 \mu \mathrm{m}$

846 thick each. Printing conditions: head speed $10 \mathrm{~mm} / \mathrm{s}$, actuation speed $38 \mu \mathrm{m} / \mathrm{s}$, printing

847 head specs: nozzle ID 254 $\mu$, linear actuator NEMA 8. Although C-shaped parts were 
848 not used in any experiments, they are included here to demonstrate the layering details.

849 Yellow bracket indicates the beveled alignment of layers. Parts in C, D, E, and F are

850 printed with a higher resolution print head (combination of nozzle diameter and linear

851 actuator properties), compared to the part in A \& B. Prints from both print heads are

852 shown for comparison. For reference, the squared-shaped prints shown in Figures $6-8$

853 were generated using the higher resolution print head, as well (see specifications in

854 Supplementary Information section). See also Supplementary Fig. 7. 
A
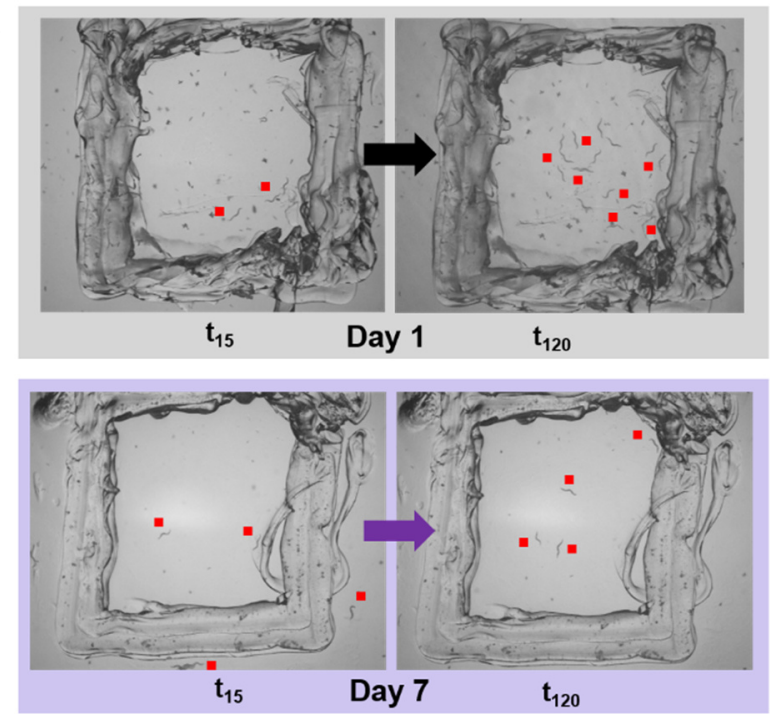

B

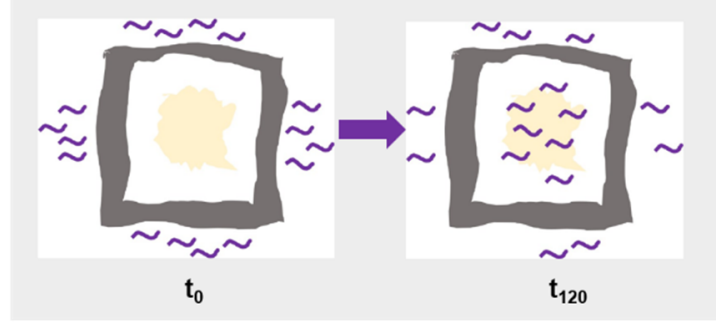

Figure 6: Assessment of C. elegans ability to cross a physical barrier to reach a food source. (A) Top panel: Snapshots of the square barrier assay when a population of adult Day 1 nematodes is tested, at $t_{0}$ (left) and $t_{120}$ (right); Bottom panel: Snapshots of the square barrier assay when a population of adult Day 7 nematodes is tested, at to (left) and $\mathrm{t}_{120}$ (right). Red dots indicate location of nematodes; number of dots does not correspond to number of nematodes. Scattered dark spots in the substrate are noticeable due to localized crystallization of NGM components, which often happens if the plates are a few weeks old (see Supplementary Information), however the overall suitability of the plate is not compromised. (B) Schematic of the assay. An NGM 3Dprinted square (gray) is placed on an NGM plate, and E. coli OP50 food (yellow) is pipetted inside the square. A population of $C$. elegans nematodes (purple lines) is nematode is inside the square (nematodes placed on assay plate). At $t_{120}$ a fraction of the worms' population has crossed the square barrier and is foraging on the food. For 
891 the control experiment, the 3D-printed square is absent, and a figurative square is

892 marked at the bottom of the plate, to define the target area (6D, left). (C) Graph showing

893 the \% of two age groups of nematodes that crossed the square barrier over 120min;

894 purple diamonds: adult Day 1 hermaphrodites, (three independent experiments, $\mathrm{n}_{1}=18$,

$895 \mathrm{n}_{2}=12, \mathrm{n}_{3}=11$ ); black circles: adult Day 7 hermaphrodites, (three independent

896 experiments, $\left.\mathrm{n}_{1}=12, \mathrm{n}_{2}=16, \mathrm{n}_{3}=16\right)$. As a control experiment, adult day 1 and adult Day

8977 nematodes were assayed on dishes with figurative square frame over 120min; pink

898 diamonds: adult Day 1 hermaphrodites, (three independent experiments, $\mathrm{n}_{1}=21, \mathrm{n}_{2}=17$,

$899 \mathrm{n}_{3}=17$ ); grey circles: adult Day 7 hermaphrodites, (three independent experiments, $\mathrm{n}_{1}=6$,

$900 \mathrm{n}_{2}=8, \mathrm{n}_{3}=9$ ); Comparisons made using multiple unpaired t-tests, $p$ values significant

901 when $p>0.05 ;{ }^{* *}: p<0.01,{ }^{* * *}: p<0.001$, only significant comparisons between final (at

$902 \mathrm{t}=120 \mathrm{~min}$ ) are shown; error bars indicate standard deviation, dashed line indicates 50\%

903 level. See also Supplementary Table S1. (D) Schematic showing the relative size and

904 position of the figurative square frame used in the control experiments (left) and of the

905 3D-printed square used in the physical barrier experiments (right), with respect to a

$90660 \mathrm{~mm}$ petri dish. 3D-printed squares were $\sim 20 \times 20 \mathrm{~mm}, 3$ layers, $0.5 \mathrm{~mm}$ thick each.

907 Brown triangles indicate initial placement of nematodes.

908

909

910

911

912

913

914

915

916

917

918

919 
A
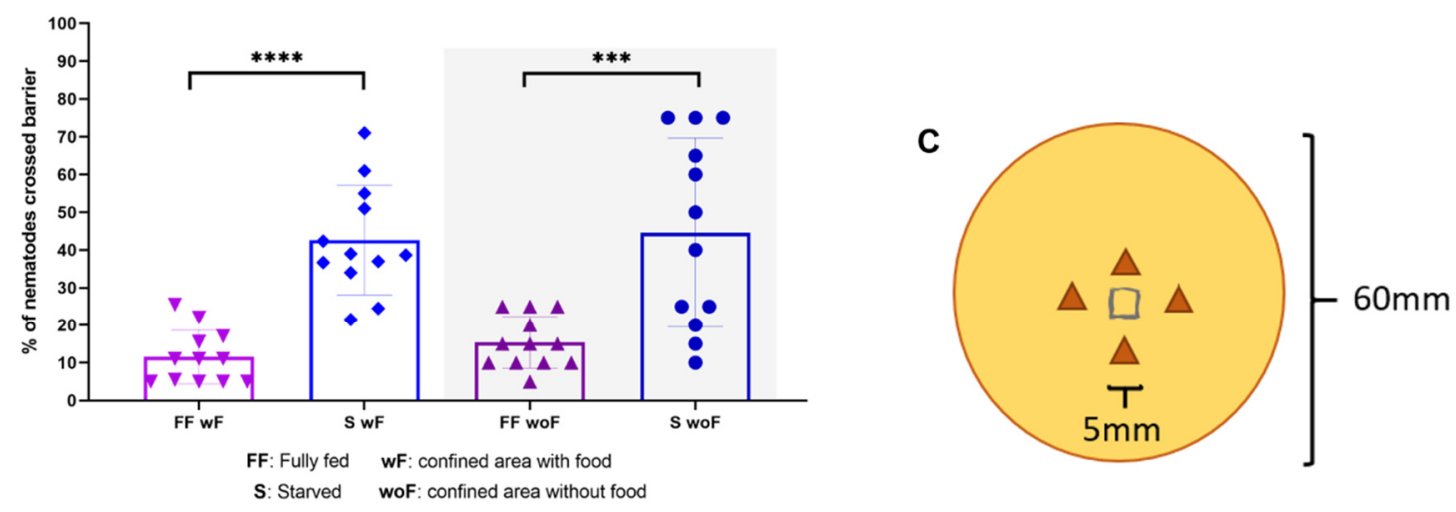

B
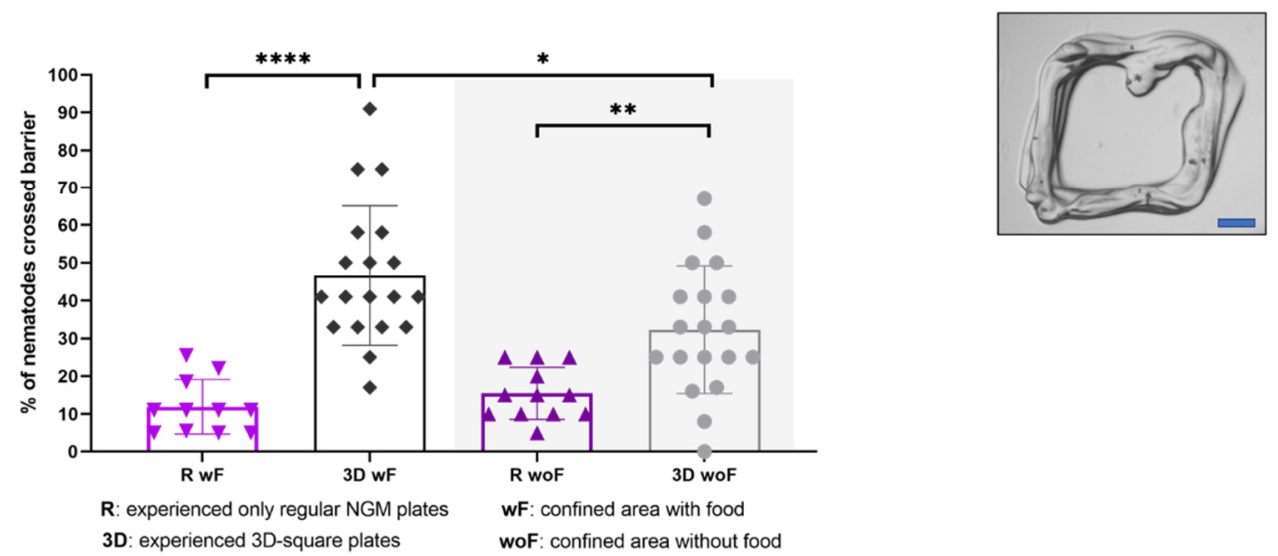

922 Figure 7: C. elegans physical barrier crossing with respect to age and feeding status. (A) Graph showing the \% of four groups of adult Day 1 nematodes that crossed

924 the square barrier over 120min; purple inverted triangles: nematodes that have been 925 fully fed (FF), cross the barrier into a square baited with food (wF); blue diamonds:

926 nematodes that have been starved (S) for 24 hours prior testing, cross the barrier into a 927 square baited with food (WF); purple triangles: nematodes that have been fully fed (FF), 928 cross the barrier into a square without food (woF); blue circles: nematodes that have been starved (S) for 24 hours prior testing, cross the barrier into a square without food

930 (woF). (B) Graph showing the \% of four groups of fully fed Day 1 adult nematodes that 931 crossed the square barrier over 120min; purple inverted triangles: nematodes that have been grown on regular NGM plates $(R)$, cross the barrier into a square baited with food

933 (WF); black diamonds: nematodes that have experienced a 3D square for $24 \mathrm{hrs}$ prior 934 testing (3D), cross the barrier into a square baited with food (WF); purple triangles:

935 nematodes that have been grown on regular NGM plates (R), cross the barrier into a 
936 square without food (woF); grey circles: nematodes that have experienced a 3D square 937 for $24 \mathrm{hrs}$ prior testing (3D), cross the barrier into a square without food (woF). Data of

938 C. elegans grown on regular NGM plates (purple triangles and inverted triangles, R) are

939 the same with fully fed nematodes data (FF) in panel $A$. Panels $A$ and $B$ : Each data

940 point corresponds to the percentage of worms scored inside the target square at a given

941 timepoint, measurements taken every 5 or $10 \mathrm{~min}$ over a $120 \mathrm{~min}$ period, horizontal lines

942 indicate the mean, error bars indicate the standard deviation. Comparisons made using

943 two-tailed, unpaired t-test, $p$ values significant when $p>0.05 ;{ }^{*}: p<0.05,{ }^{* *}: p<0.01,{ }^{* * *}$ :

$944 p<0.001,{ }^{* * *}: p<0.0001$, only significant comparisons shown. Shaded area: confined

945 area did not contain food (without food: woF). (C) Top: Schematic showing the relative

946 size of the $5 \times 5 \mathrm{~mm} 3 \mathrm{D}$-printed square with respect to a $60 \mathrm{~mm}$ petri dish, brown triangles

947 indicate initial placement of nematodes. Bottom: Example of a 3D-printed square used

948 in the above experiments, $5 \times 5 \mathrm{~mm}, 3$ layers, $0.5 \mathrm{~mm}$ thick each; scale bar: $1 \mathrm{~mm}$. 

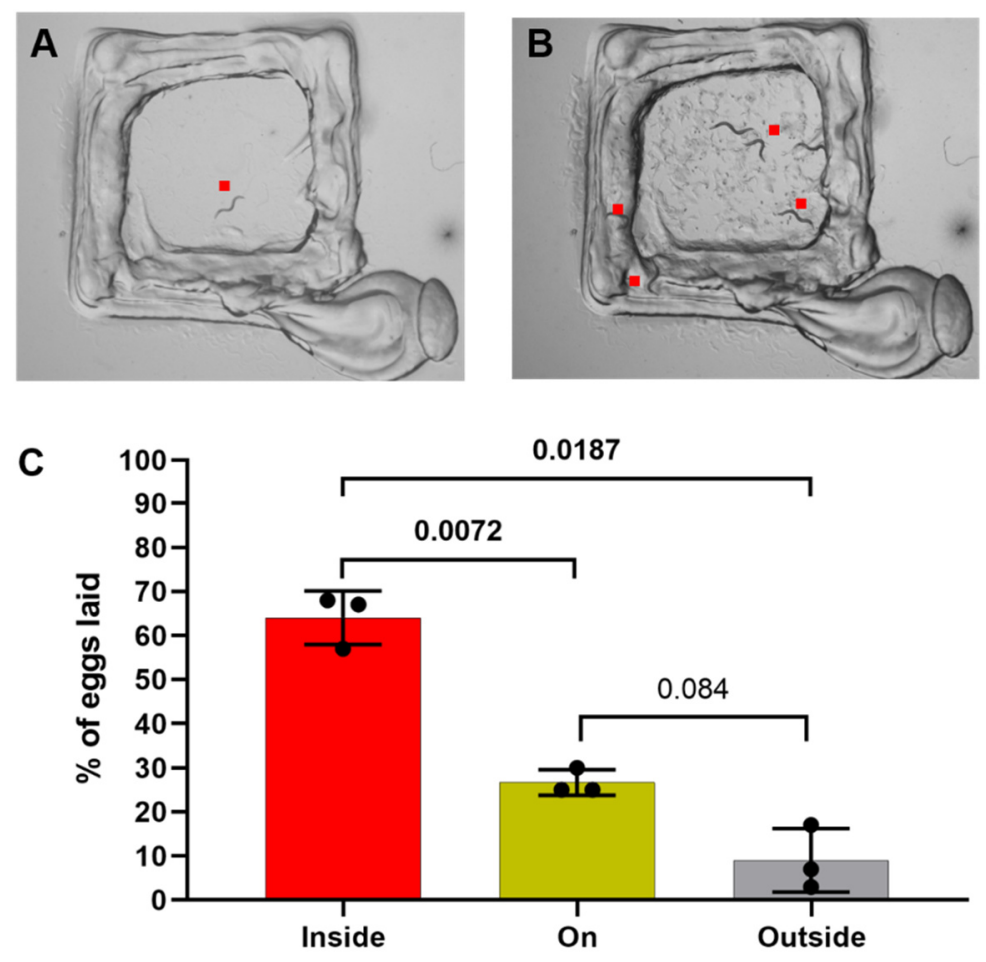

Figure 8: 3D-printed structures can be used to confine $C$. elegans behavior. (A) 3D-printed square $(5 \times 5 \mathrm{~mm}, 3$ layers, $0.5 \mathrm{~mm}$ thick each), seeded with OP 50 , on an NGM plate, at time $t=0$. (B) The same square as in $A$, after 24 hours ( $t=24 h r)$. Eggs have been laid only inside the confined area, and several nematodes can be seen on and inside the square. Note the absence of eggs in the proximity of the square frame. Nematodes initially placed as in Fig. 7C. (A) and (B): red dots indicate location of $C$.

971 elegans. (C) Graph showing the \% of eggs laid in the region of each 3D square tested,

972 as shown in A and B, inside: eggs laid inside the framed area, on: eggs laid on the

973 square frame, outside: eggs laid in the premises of the squares, in $5 \mathrm{~mm}$ distance. Bars:

974 mean, error bars: SD, dots: 3 individual experiments (worms used in each experiment:

$975 n_{1}=10, n_{2}=12, n_{3}=11$ ), above bars: $p$-values of indicated comparisons, student's paired t976 test, with bold $p$-values<0.05 (significant difference). 


\section{Supplementary Figures and Tables}

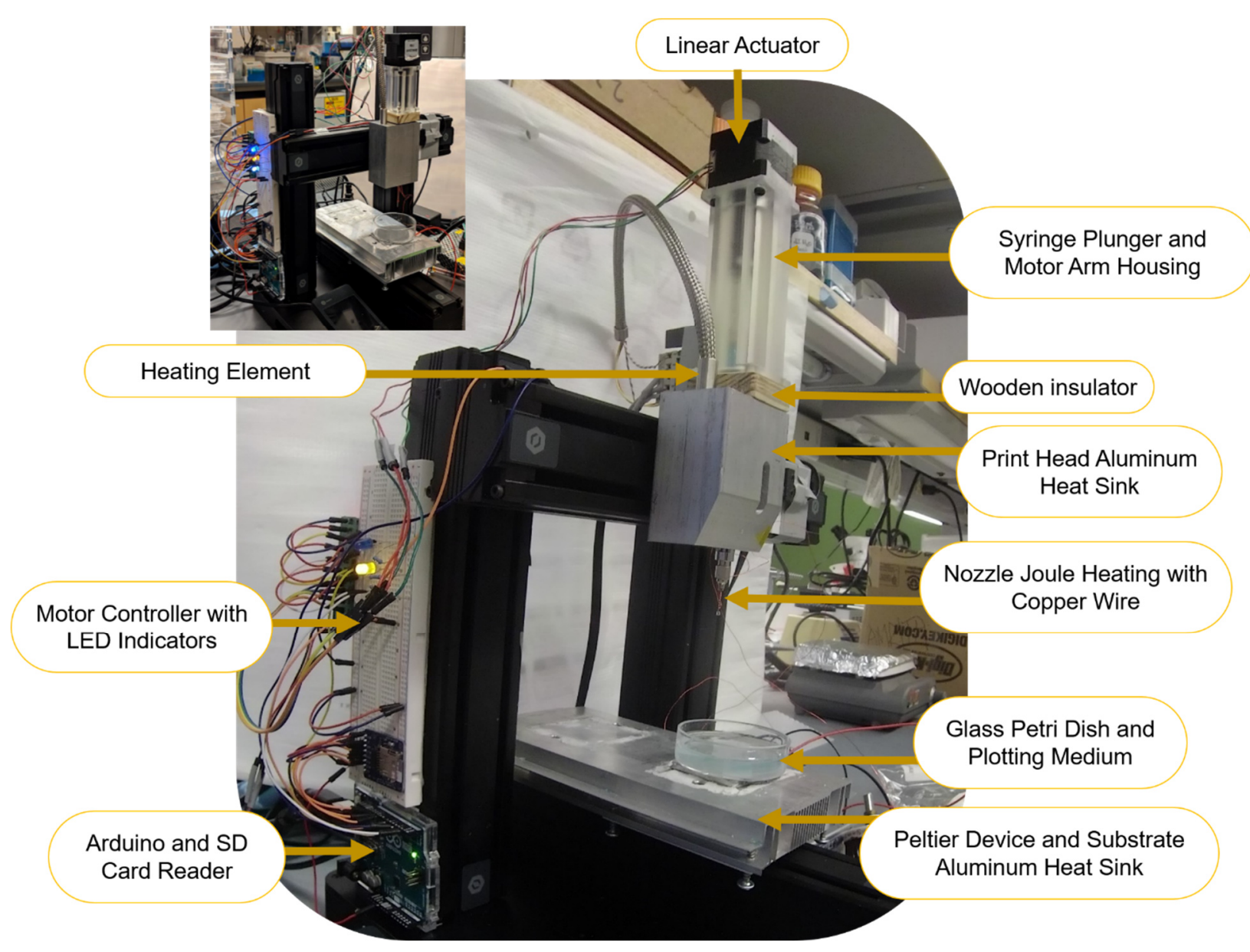

983 Supplementary Figure S1: Overview of the Parnon printer. The major customized and house-made parts are indicated. Small inset: early version of the Parnon printer, in which a shorter linear actuator and syringe housing are featured. 

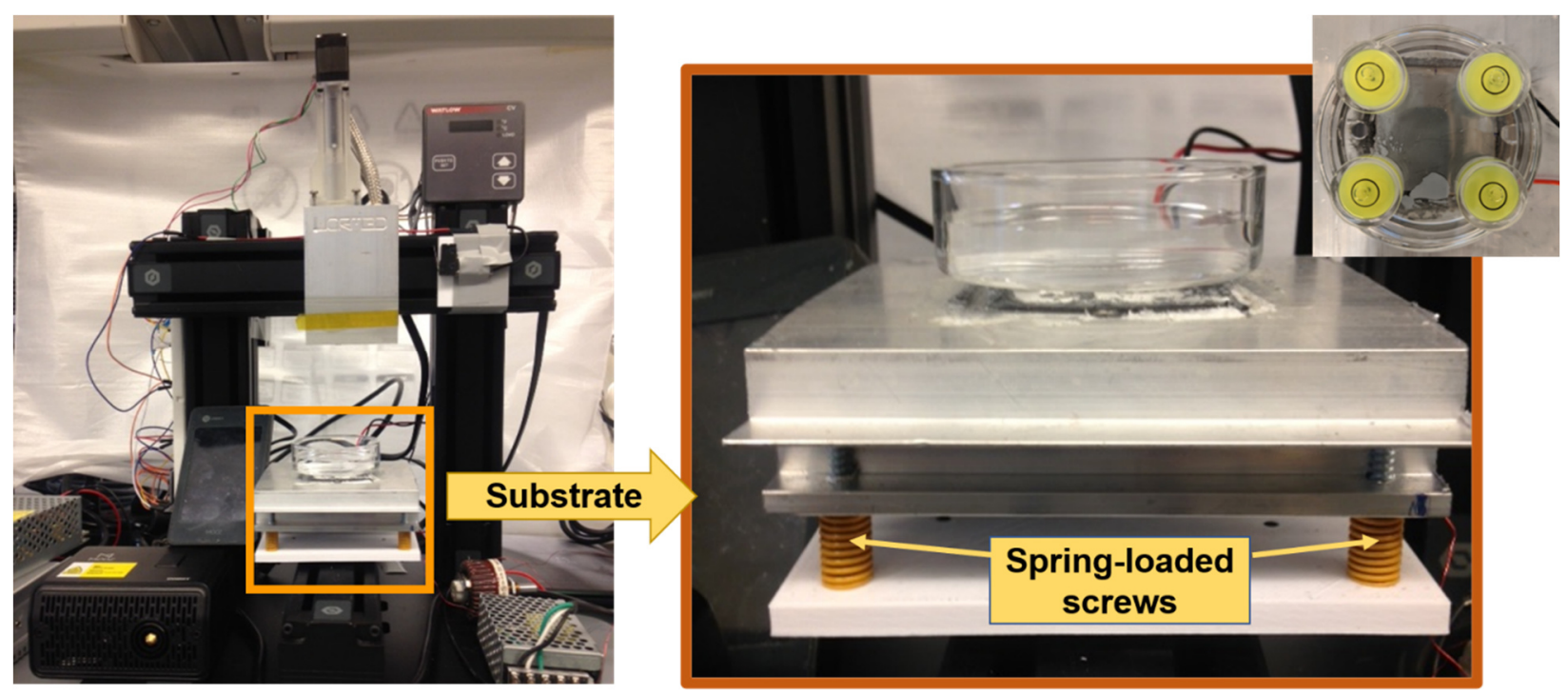

994

995 Supplementary Figure S2: Four-point bed leveling system. Each corner of the

996 substrate is equipped with a spring-loaded screw, to either raise or lower the

997 corresponding corner of the substrate. Circular levels are placed at each corner of the

998 substrate (small inset) and the screws are adjusted until each level reading matches the 999 rest. 

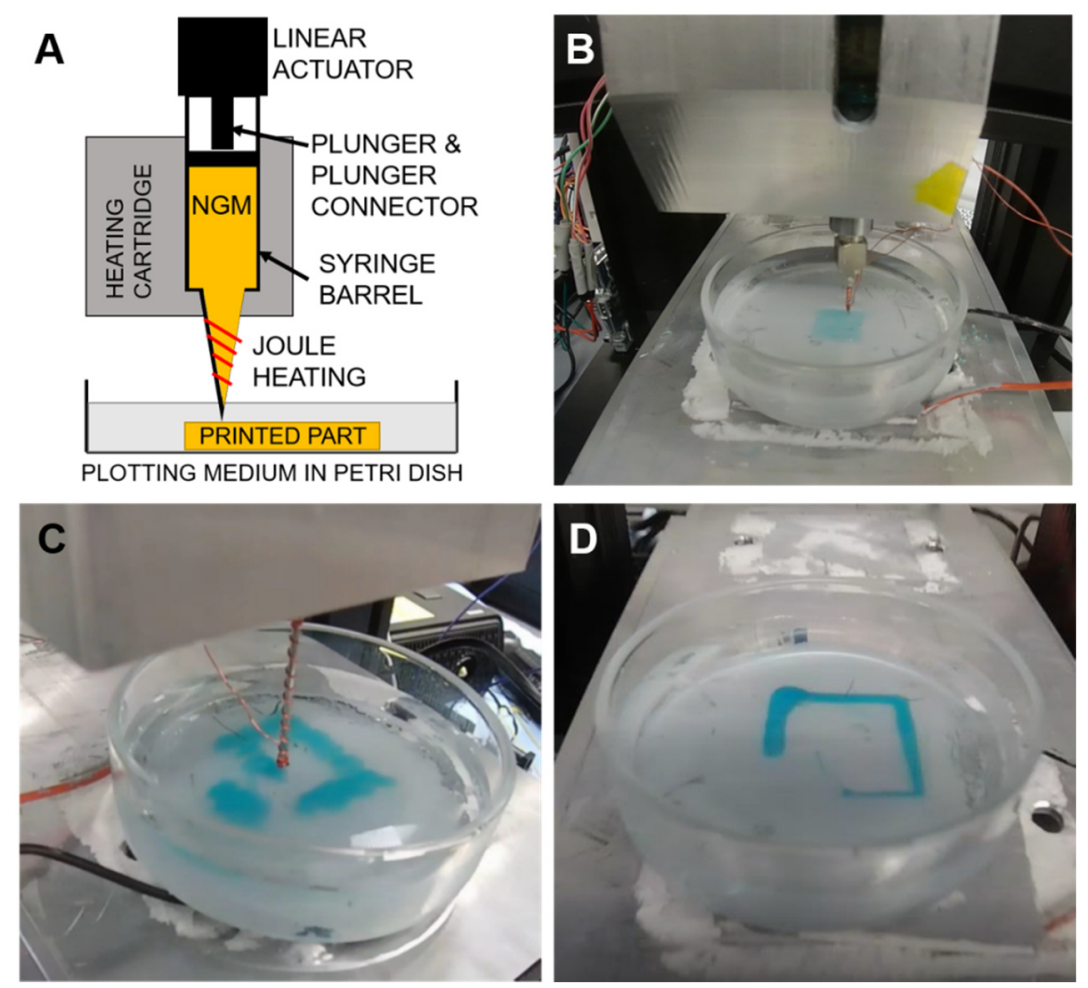

Supplementary Figure S3: The printing apparatus and the nozzle joule heating

detail. (A) Schematic of the syringe barrel containing liquid NGM, sitting inside the

heating sink which contains the heating cartridge. The joule heating is illustrated around Printing a square structure; note the wider nozzle which results in thicker printed lines, result of the narrower nozzle used. 
A

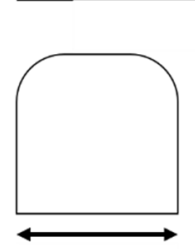

$W_{i}$
Time

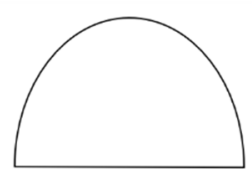

-Accel. -Decel.

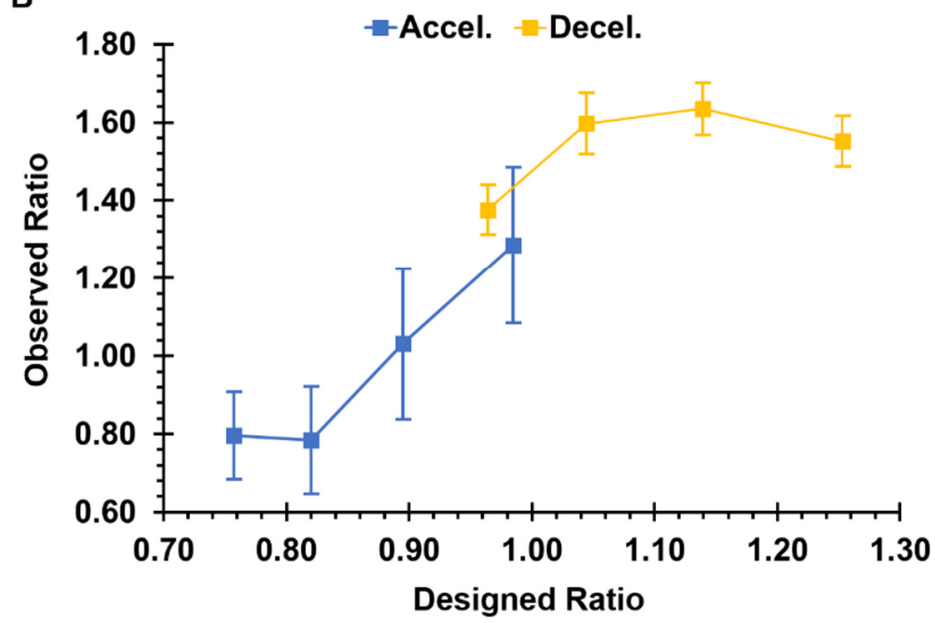

Designed Ratio
C

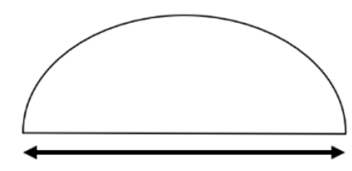

$w_{f}$

\begin{tabular}{|c|c|c|c|}
\hline \multirow{2}{*}{$\begin{array}{c}\text { Head } \\
\text { Speed }\end{array}$} & $\begin{array}{c}\text { Designed } \\
\text { Ratio }\end{array}$ & \multicolumn{2}{|c|}{ Observed Ratio } \\
\cline { 3 - 4 } & Mean & Std. Err. \\
\hline 10 & 0.76 & 0.8 & 0.11 \\
\hline 11 & 0.82 & 0.78 & 0.14 \\
\hline 12 & 0.9 & 1.03 & 0.19 \\
13 & 0.98 & 1.29 & 0.2 \\
\hline \multicolumn{4}{|c|}{ Deceleration } \\
\hline 13 & 0.96 & 1.38 & 0.06 \\
12 & 1.04 & 1.60 & 0.08 \\
11 & 1.14 & 1.63 & 0.07 \\
10 & 1.25 & 1.55 & 0.06 \\
\hline \multicolumn{4}{|c|}{} \\
\hline
\end{tabular}

Supplementary Figure S4: Liquid spreading control and line width experiments.

(A) Schematic of the cross section of a printed line that exhibits liquid spreading, evolving over time. The initial width $\left(W_{i}\right)$ is the line width immediately after the line is extruded, which is approximately equal to the width of the nozzle. As time passes, the width of the line increases while the height decreases. The final width $\left(W_{f}\right)$ is the line width after the equilibrium shape has been reached. (B) Graph showing the relationship between the ratios of designed line widths and observed line widths to $W_{i}$. A designed ratio of 1 means the expected value for $W_{f}$ is equal to the width of the nozzle. The designed line width is the expected value for $W_{f}$ based on user defined extrusion volume. An observed ratio of 1 means the final width $\left(W_{f}\right)$ is equal to the width of the nozzle. The observed line width is the measured value for $W_{f}$ after printing. Means with standard error bars are shown. To achieve an observed ratio of 1 ( $W_{f}=$ nozzle width) the design ratio needs to be $\sim 0.90$. Table shows data from line width experiments. (C) 
1032 Image from the line width experiments. Four consecutive $20 \mathrm{~mm}$ long lines were printed 1033 with a head speed ranging from $10 \mathrm{~mm} / \mathrm{s}$ to $13 \mathrm{~mm} / \mathrm{s}$. In four experiments, the head 1034 speed started at $10 \mathrm{~mm} / \mathrm{s}$ and incremented $1 \mathrm{~mm} / \mathrm{s}$ per line with an actuation speed of $103513.4 \mu \mathrm{m} / \mathrm{s}$. In another four experiments, the head speed started at $13 \mathrm{~mm} / \mathrm{s}$ and 1036 decremented $1 \mathrm{~mm} / \mathrm{s}$ per line with an actuation speed of $17.0 \mu \mathrm{m} / \mathrm{s}$. The software that 1037 accompanies the microscope used to take these pictures allows for measurements in 1038 pixels and subsequent conversion to $\mathrm{mm}$. Scale bar: $2 \mathrm{~mm}$. 


\section{A}

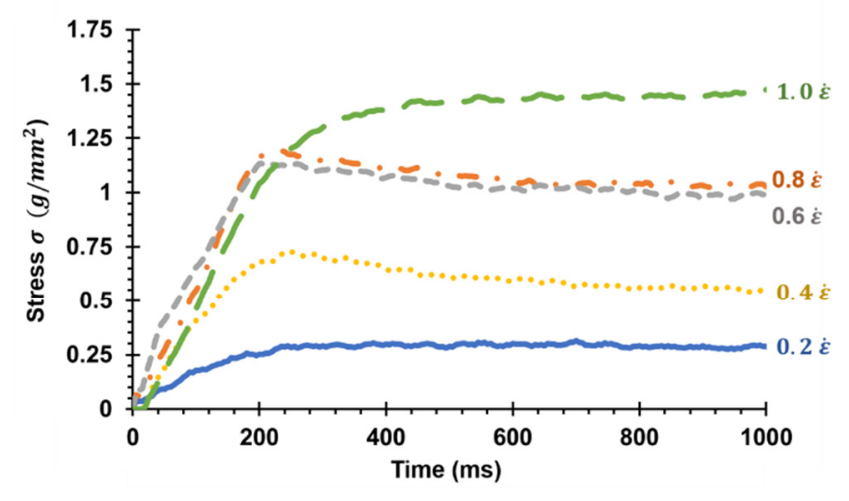

C

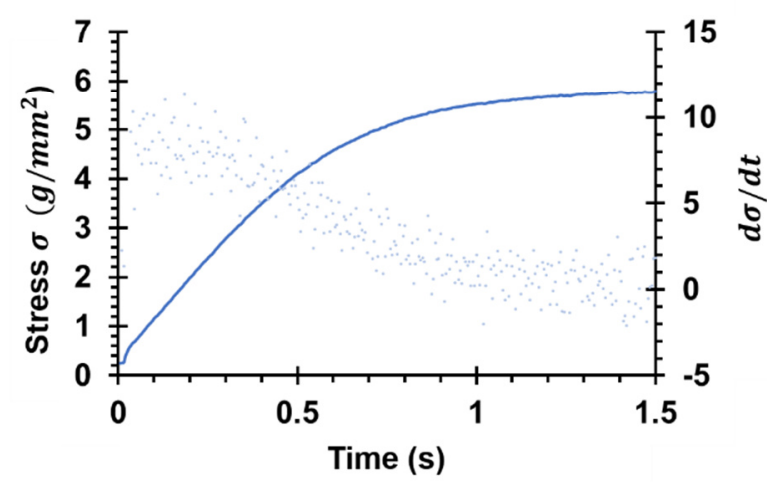

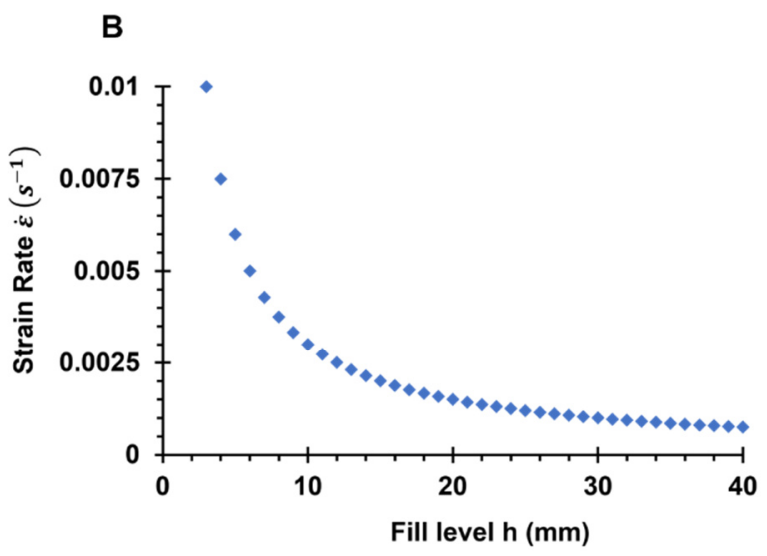

D

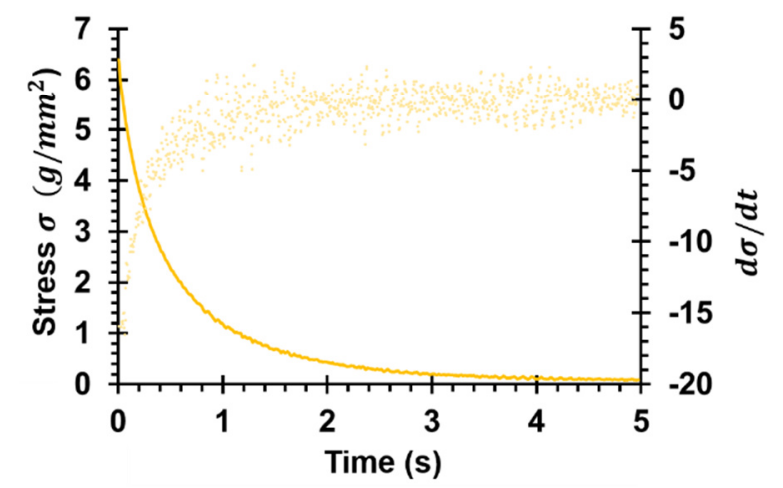

Supplementary Figure S5. The viscoelasticity of NGM. The compressive strain rate is directly proportional to the equilibrium stress in the liquid NGM during extrusion. (A) Graph showing how stress $\sigma$ changes over time, for 5 different strain rates $\dot{\varepsilon}\left(s^{-1}\right)$. NGM extruded at a constant actuation speed will result in increasing strain rates (see also Supplementary Fig. S5). (B) Strain rate varies with the NGM fill level in the syringe and the actuation speed. Study done with $30 \mu \mathrm{m} / \mathrm{s}$ actuation speed. The syringe used in the Parnon is considered full at 50 linear millimeters of NGM. Eq. SF4 describes the inverse relationship of strain rate $(\dot{\varepsilon})$ to fill level $(h)$ given a constant actuation speed (v). Eq. SF4: $\dot{\varepsilon}=\frac{h}{v}$. The values for strain rate $(\dot{\varepsilon})$ are unique to the syringe used in the 1070 Parnon, but the trend is expected to apply in all cases of actuation pressure extrusion. 1071 (C): Comparison of increasing $\sigma$ (continuous line, left y axis) and $d \sigma / d t$ (scatter plot, 1072 right y axis) over time (stress build up). When $d \sigma / d t$ reaches 0 , the inflection point has 
1073 been reached. The time it takes for the inflection point to be reached is $t_{\text {eq. }}$ (D):

1074 Comparison of decreasing $\sigma$ (continuous line, left y axis) and $d \sigma / d t$ (scatter plot, right y 1075 axis) over time (stress relaxation). When $d \sigma / d t$ reaches 0 , the inflection point has been 1076 reached. The time it takes for the inflection point to be reached is $t_{r}$. It was found that $1077 t_{e q .}=t_{r}=\sim 600 \mathrm{~ms}$ at $\dot{\varepsilon}=1.1$. Panels $\mathrm{C}$ and $\mathrm{D}$ : Compressive results using a $5 \mathrm{~mL}$, $107812 \mathrm{~mm}$ ID syringe and a 404 ID nozzle.

1079

1080

1081

1082

1083

1084

1085

1086

1087

1088

1089

1090

1091

1092

1093

1094

1095

1096

1097

1098 

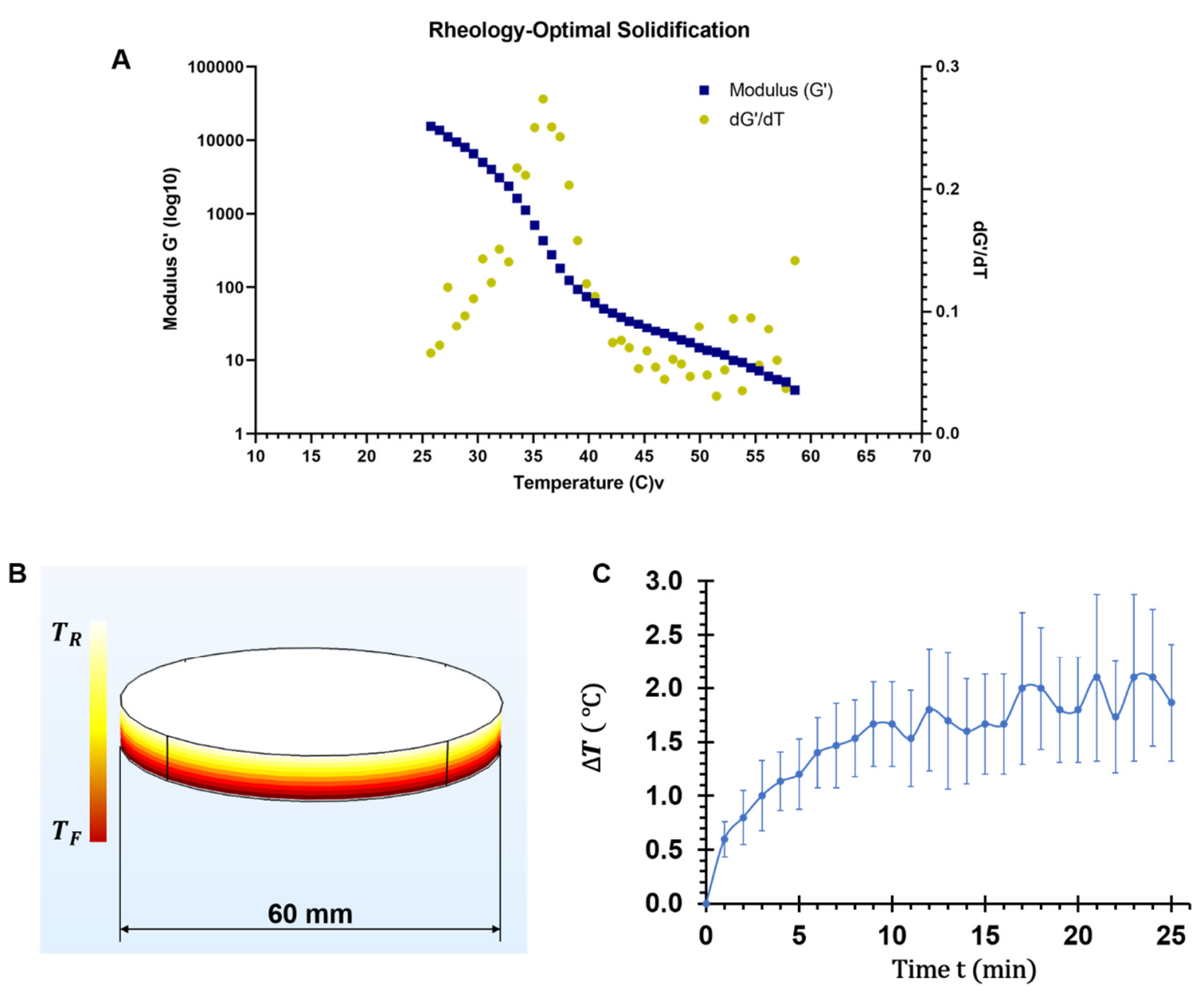

Supplementary Figure S6: NGM solidification rheology, and heat flux model of the

substrate. (A) Graph shows the storage modulus G' (blue squares, left y axis) and rate of change $\mathrm{dG}^{\prime} / \mathrm{dT}$ (green circles, right y axis) as a function of temperature. The highest value of $\mathrm{dG} / \mathrm{dT}$ corresponds to $35.9^{\circ} \mathrm{C}$, meaning that at this temperature solidification occurs the fastest. Rheology experiment performed on a TA DHR2 Rheometer at a cooling rate of $5{ }^{\circ} \mathrm{C} / \mathrm{min}$ and an angular frequency of $1 \mathrm{rad} / \mathrm{s}$. (B) COMSOL visualization of the temperature gradient across the plotting medium (plotting medium cylinder volume: $20 \mathrm{~mL}$ ). The cooling source is the heat flux (Q) provided by the Peltier device beneath the substrate. The temperature drop $(\Delta T)$ is defined as $\Delta T=T_{R}-T_{F}$, where $T_{R}=25{ }^{\circ} \mathrm{C}$ (estimated room temperature) and $T_{F}$ is dependent on the effective heat flux (Q). (C) Actual temperature drop over time, with mean of three independent experiments and standard error bars. The cooling levels out at a $\Delta \mathrm{T}$ of $\sim 2.0^{\circ} \mathrm{C}$. Using 
1113 Eq. F9 our Peltier device is achieving an effective heat flux of $182 \mathrm{~mW} / \mathrm{cm}^{2}$ or $\sim 5.15 \mathrm{~W}$

1114 over the entire $60 \mathrm{~mm}$ petri dish. Eq. F9 describes the linear relationship between

1115 Temperature Reduction $\left(\Delta \mathrm{T}\right.$, in $\left.{ }^{\circ} \mathrm{C}\right)$ and Heat Flux $\left(\mathrm{Q}\right.$, in $\left(\mathrm{mW} / \mathrm{cm}^{2}\right)$.

1116 Eq. F9: $\Delta T=0.011 Q$

1117

1118

1119

1120

1121

1122

1123

1124

1125

1126

1127

1128

1129

1130

1131

1132

1133

1134

1135

1136

1137

1138 

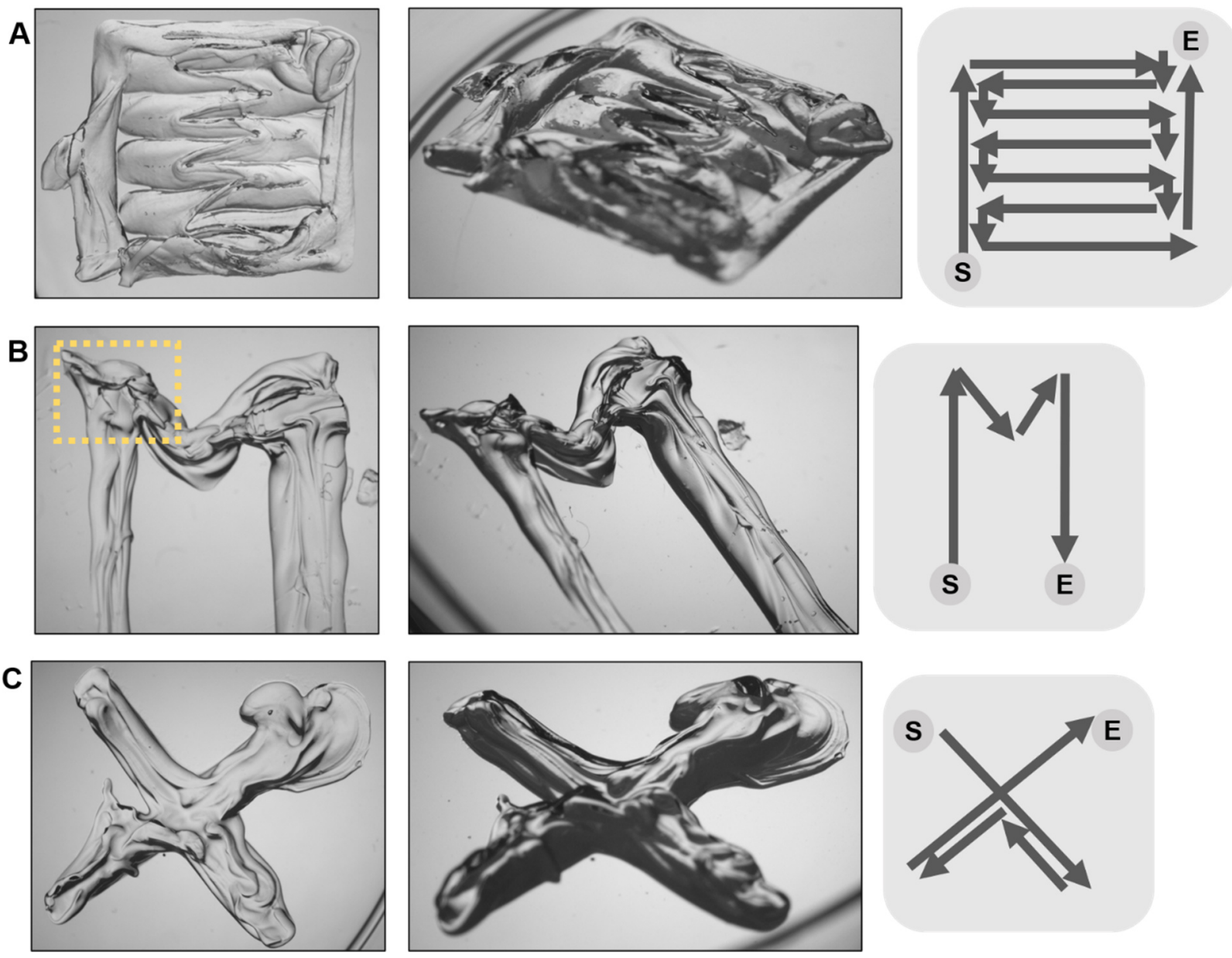

Supplementary Figure S7: Parnon-printed structures and printing paths. These structures were printed with a larger diameter nozzle $(400 \mu \mathrm{m}-1 \mathrm{~mm}$ ID) than the structures in Fig 5. (A) NGM pad, printed to test Parnon's ability to create continuous

1144 sheets. (B) M-shaped print, to test Parnon's ability to lay NGM lines in acute $\left(<90^{\circ}\right)$ angles, yellow frame indicates reduced printing quality area. (C) A cross-shaped print, to test Parnon's ability to print cross-shaped designs, without having to pause extrusion,

1147 relocate, and restart extrusion. All panels: Left: top view; middle: perspective view; right: 1148 schematic of printing path; S: start, E: end. 

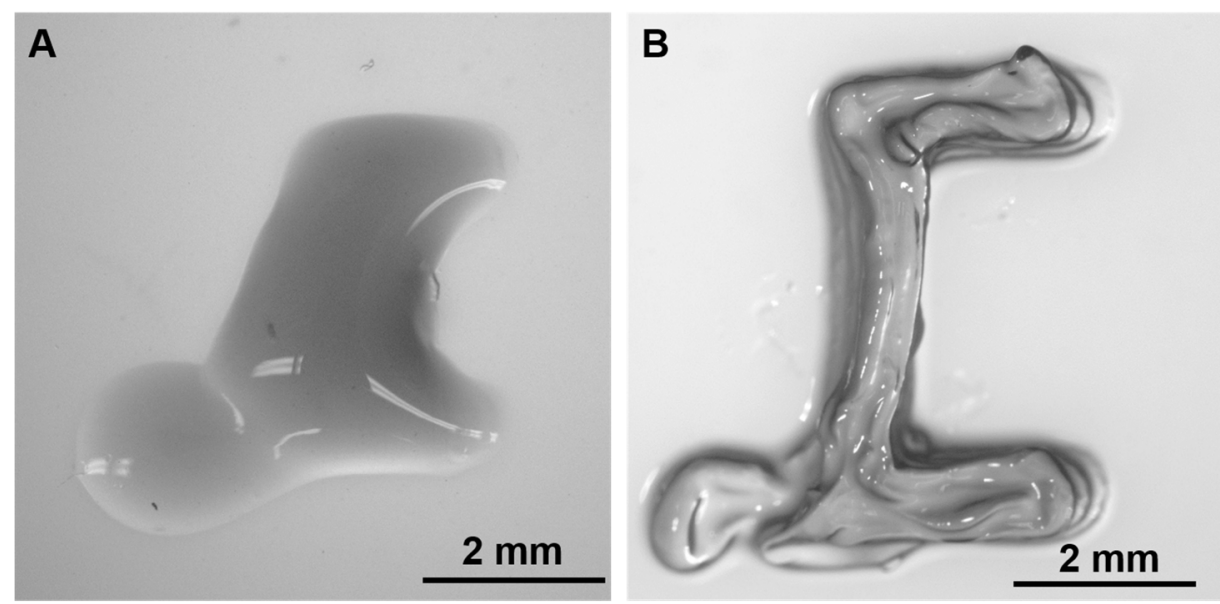

1155 Supplementary Figure S8: Printed part quality comparison with respect to plotting medium usage. (A) C-shaped part printed without plotting medium, exhibiting extensive liquid spreading, top view. (B)

(B) Same C-shaped design printed using plotting 1158 medium, with minimal liquid spreading, top view. This is the same C-shaped part as in 1159 Figure 5A \& 5B. 

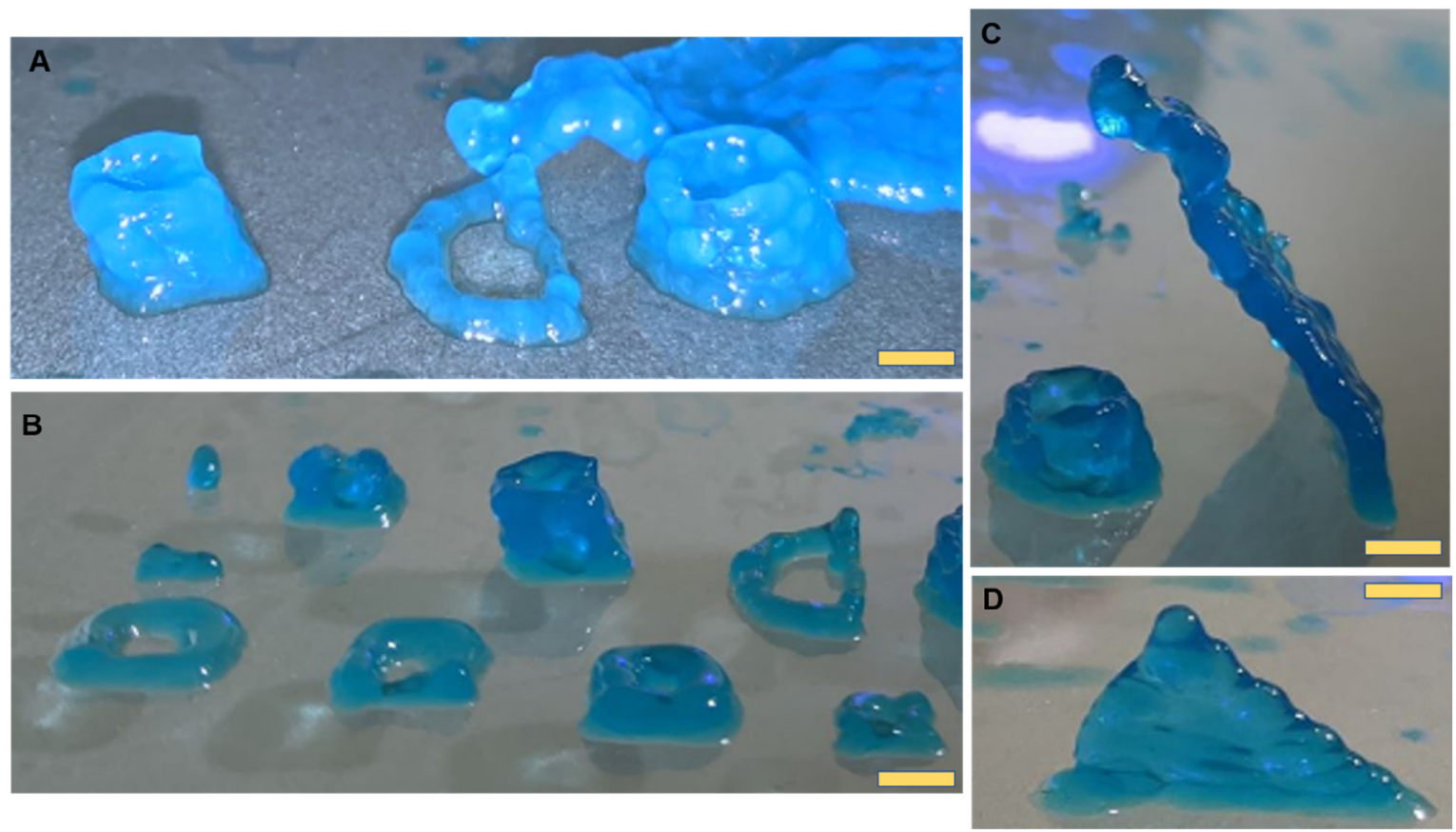

Supplementary Figure S9: Printing attempts for multi-layer NGM parts. (A)

Examples of 1- to 4-layer cylindrical structures, scale bar: $5 \mathrm{~mm}$. (B) Examples of 1- to

5-layer cylindrical structures, front row printed in the absence of plotting medium, scale bar: $6 \mathrm{~mm}$. (C) A 5-layer cylinder and a 14-layer triangular tilted wall (side view), scale bar: $6 \mathrm{~mm}$.

(D) A12-laye view), scale bar: $3 \mathrm{~mm}$. Blue dye: food color (Americolor, USA). 


\section{Supplementary Information}

1191 A full list of the major parts and items used for the customization of Parnon printer is 1192 provided in the Appendix. The Arduino code is available upon request.

\section{i. Preparation of $3 D$ printed structures for $C$. elegans experiments}

After printing, the 3D parts are removed from the plotting medium and are placed on an unseeded NGM plate. If they are to be used immediately, i.e. in the next 24 hours, they are rinsed five times with deionized water, then are left to dry off excess moisture, in sterile conditions. If they are to be used in longer than 24 hours, then they are washed with $5 \%$ sodium hypochlorite, for $15 \mathrm{~min}$ on an orbital shaker, followed by five rinses with deionized water, 10 min on the orbital shaker, each. This precaution is necessary since the parts are not printed in sterile environment. Next, the plates are left

1202 to dry off excess moisture in sterile conditions. The plates can be sealed with parafilm and stored in $4^{\circ} \mathrm{C}$ for several days, in which case they need to reach room temperature before use. If the plates are used after a few weeks, some evidence of crystallization 1205 might be noticeable in the plate NGM (see for example Fig. 6D and in less extent 6E), 1206 but the overall suitability is not compromised.

\section{ii. Actuation pressure}

The linear actuator (Fig. 2 Part $\mathrm{Ci}$ ) is a NEMA-8 captive with a 1: 1 step stroke of

$12103 \mu \mathrm{m}$. We use a 1:4 step ratio for an effective step stroke $\left(l_{s t}\right)$ of $0.75 \mu \mathrm{m}$. The actuator 1211 has a $38 \mathrm{~mm}$ total stroke length and a volume capacity of $2,470 \mathrm{~mm}^{3}$. A typical part 1212 design will utilize a volume of $50 \mathrm{~mm}^{3}$. Parnon's capacity is roughly $50 x$ the average 1213 print volume. It is powered by a 40V power supply through a TB6600 Stepper Motor 1214 Driver. The motor driver has microstepping capability, a 1:2 step stroke is then $1.5 \mu \mathrm{m}$.

1215 The linear actuator is rated for to run at $0.49 \mathrm{~A}$, the motor driver is set to $0.5 \mathrm{~A}$. This 1216 linear actuator was chosen because it has the highest stroke precision the manufacturer 1217 could offer. An early version of Parnon utilized a NEMA-14 linear actuator that had a 1218 lower stroke precision. A higher stroke precision results in higher quality prints. The 1219 parts in Figs. 5C, 5D, 5F, and 6 are printed with the early version of Parnon, wearing 
1220 NEMA-14; the parts in Figs. 5A, 5B, 5E, 7, and 8 are printed with the recent version of 1221 Parnon, featuring NEMA-8.

$1222 \quad$ The Arduino card communicates with the motor driver by sending $5 \mathrm{~V}$ pulses in a 1223 square wave. A user defined delay time $\left(t_{d}\right)$ is how long the pulse is sustained for. Each 1224 pulse equates to one step taken by the linear actuator. A total print time $\left(t_{p r}\right)$ is derived 1225 during the gCode writing process. The total number of steps, or pulses, during a print 1226 control the extruded volume of NGM. A theoretical extruded volume $\left(V_{\text {ex. }}\right)$ can then be 1227 calculated.

$$
\text { Eq. 1: } \quad V_{e x}=\frac{l_{s t} t_{p r}}{2 t_{d}}
$$

By varying $t_{d}, V_{e x}$ can be controlled. A lower $t_{d}$ will result in a more fluent stepping process in the actuator and higher $V_{p r}$.

The gCode defines the layer height $\left(h_{l}\right)$ and the print path length $\left(l_{p r}\right)$. Using the nozzle ID $\left(w_{i}\right)$, a theoretical print volume $\left(V_{p r}\right)$ can be calculated.

$$
\text { Eq. 2: } \quad V_{p r}=h_{i} l_{p r} w_{i}
$$

We refer to the designed ratio of the print as the ratio of $V_{e x}$ to $V_{p r}$. Despite the Parnon's several features meant to limit the amount of liquid spreading, some amount of liquid spreading still occurs. A print quality comparison between prints that resulted while the liquid spreading mitigations were active vs when the plotting medium and cooling effect were not used is presented in Supplementary Figure 4.

The amount of liquid spreading the fully functioning Parnon experiences was studied so the actual width of a line of printed NGM becomes predictable and designable. Results of the line width experiment are shown in Supplementary Figure 1.

1242 Four $20 \mathrm{~mm}$ long lines of NGM were printed, with $5 \mathrm{~mm}$ transitions. Two experiments, 1243 accelerating and decelerating, were repeated 3 times each. A relationship between the 1244 designed ratio $\left(V_{e x} / V_{p r}\right)$ and the actual ratio $\left(w_{l} / w_{i}\right)$ of the line width $\left(w_{l}\right)$ to the nozzle 1245 ID $\left(w_{i}\right)$. A designed ratio of $\sim 0.9$ equates to an actual ratio of 1.0 (Fig. 3 ). 
1248

setting $V_{e x} / V_{p r}=0.9$ we expect $w_{l} / w_{i}=1$, or, the final line width after liquid spreading will be equal to the inner diameter of the nozzle.

\section{iii. NGM compressive viscoelastic response}

NGM in the $9.11 \mathrm{~mm}$ ID syringe used in the latest version of the Parnon was tested at strain rates starting at $\dot{\varepsilon}=0.2$, incrementing by 0.2 five times ending at $\dot{\varepsilon}=1.0$ (Fig. 4 Part A). $\sigma_{e q}$ ranged from $\sim 0.25 \mathrm{~g} / \mathrm{cm}^{2}$ at $\dot{\varepsilon}=0.2$ to $\sim 1.5 \mathrm{~g} / \mathrm{cm}^{2}$ at $\dot{\varepsilon}=1.0$.

Strain rates decrease inversely as the fill level in the syringe increases for a constant actuation speed [Suppl. Fig. 5]. In the $9.11 \mathrm{~mm} \mathrm{ID} \mathrm{syringe} t_{\text {eq. }}=\sim 300 \mathrm{~ms}$.

NGM in the $12 \mathrm{~mm}$ ID syringe used in the early versions of the Parnon was tested 4 times. We found that $t_{\text {eq. }} \cong 600 \mathrm{~ms}$ at a $\dot{\varepsilon}=1$.1. (Fig. 4 Part Bi). In the $12 \mathrm{~mm} \mathrm{ID}$ syringe $t_{e q .}=\sim 600 \mathrm{~ms}$. We believe the larger ID of this syringe caused the higher $t_{e q}$. and $\sigma_{e q}$. when compared to the lower syringe ID $(9.11 \mathrm{~mm})$ in the current version of the Parnon.

It was found that $t_{e q}=t_{r}$. The stress in the NGM during extrusion will increase with both the syringe ID and the strain rate $(\dot{\varepsilon})$. The time it takes to reach the inflection point increases with the syringe ID but is effectively constant across multiple strain rates. In practice, we found that $t_{e q}=700 \mathrm{~ms}$ with the $9.11 \mathrm{~mm}$ ID syringe results in the most accurate extrusion timing.

The time $t_{e q}$ it takes for the compressive stress $\sigma_{e q}$ in the NGM to reach a high enough level to begin pushing a relatively viscous liquid through a nozzle with a small ID $(254 \mu \mathrm{m}-400 \mu \mathrm{m})$ increases with strain rate. As part of the work done on optimizing Parnon there is a feature written in the code that automatically uses $t_{e q}$ to build up to $\sigma_{e q}$ immediately before beginning extrusion during a print. With this addition the Parnon begins extruding NGM at the right time. Before, the NGM often extruded late as time had to pass for $\sigma_{e q}$ to be reached. We speculate that the NGM near the nozzle where the resulting curves rose above $\sigma_{e q}$ before approaching it ( $\left.\dot{\varepsilon}=0.4,0.6 .0 .8\right)$ were more viscous and therefore required more stress to be extruded. In addition, $\sigma_{e q}$ for $\dot{\varepsilon}=$ $0.6,0.8$ are relatively close in value. We believe this occurs because of differing viscosity conditions in the NGM during extrusion. 
iv. gCode and software communication

Modifying the hardware of an existing 3D printer compromised the communication the printer had with the original print head. To resolve this issue, we introduced a limit switch, an Arduino card, and a stepper motor driver. limit switch, thus signifying to the software that the print has begun.

An Arduino Uno board connected to a Stepper Motor Driver which in turn is connected to the linear actuator. A custom Microsoft Excel program that processes raw 1287 cartesian coordinates and head speed values outputs three essential printer inputs:

1288 a) A gCode file that the DOBOT MOOZ-2 will interpret into XYZ axis actuation commands. The gCode file is loaded on a USB external drive or a micro SD card and 1290 placed in the appropriate slot on the Parnon.

b) The time between the custom print head hitting the limit switch and beginning actuation pressure. We refer to this value as delay one (delay1) and it is measured in 1293 milliseconds.

c) An array of step delays (measured in milliseconds), steps to take and the relative volume (Designed Ratio Fig. 3 Part B). The step delay $\left(t_{d}\right)$ and number of steps $(n)$ 1296 which correspond to a designed ratio of $\sim 0.90$ are inputted through the Arduino serial 1297 port.

Outlining the part is currently done in a custom Microsoft Excel program. Each coordinate $\mathrm{XYZ}$ that the print head will travel to during the print is individually assigned to a row in order. Upon executing the custom Microsoft Visual Basic program, the coordinates are processes and written into a gCode file. The gCode is processed by the existing firmware from DOBOT.

Time needs to pass between the print head hitting the limit switch and the point 1304 at which extrusion should begin. This time is referred to as delay one (delay1), and 1305 depending on the part design varies around $2.4 \mathrm{~s}$. The viscoelastic response NGM has 1306 to compressive stress requires a significant amount of time $\left(t_{e q .}=700 \mathrm{~ms}\right)$ to pass 1307 before sufficient stress has been reached for extrusion to commence. $t_{e q}$. is included in 
1308 1309

delay 1 and is referred to as strain in the Arduino Code. Section iv of Supplementary Information refers in detail to NGM's response to compressive stress.

The total print time $\left(t_{p r}\right)$ is derived in the Excel program as a result of dividing the path length $\left(l_{p r}\right)$ by the head speed $\left(v_{h}\right) . l_{p r}$ and $v_{h}$ are defined in the gCode.

Depending on the delay time between each step $\left(t_{d}\right)$, the number of steps for the actuator to take $(n)$ so it stops extruding when the print stops can be calculated.

$$
\text { Eq.3: } \quad n=\frac{t_{p r}}{2 t_{d}}
$$

Depending on the distance each actuator step is $\left(l_{s t}\right)$, discussed in more detail in the next section, there will be a specific calculatable volume for each selection of $t_{d}$. Multiplying the cross-sectional are of the syringe $\left(A_{s}=65 \mathrm{~mm}^{2}\right)$ by the distance the actuator will travel during the print allows the theoretical total extruded volume $\left(V_{e x}\right)$ to be calculated.

$$
\text { Eq. 4: } V_{e x}=A_{s} n l_{s t}
$$

Comparing this theoretical volume to the total print volume $\left(V_{p r}\right)$ gives us what we refer to as the designed ratio $\left(V_{e x} / V_{p r}\right)$. How this value compares to what can actually be observed and is discussed in detail in the next section.

\section{v. Plotting medium}

A COMSOL Multiphysics simulation was ran on $20 \mathrm{~mL}$ of plotting medium in a $60 \mathrm{~mm}$ diameter cylinder (Fig. 5 Part C). A negative heat flux was placed on the bottom of the geometry to model the cooling effect of the Peltier device. The relationship between the heat flux $\left(Q, \mathrm{~mW} / \mathrm{cm}^{2}\right)$ and the temperature drop across the plotting medium $\left(\Delta T,{ }^{\circ} \mathrm{C}\right)$ is described by $E q .5$.

$$
\text { Eq.5: } Q=90.91 \Delta T
$$

It was determined experimentally that after $\sim 20 \mathrm{~min}, \Delta T=\sim 2.0^{\circ} \mathrm{C}$ (Fig. 5 Part D). As a result of the COMSOL modeled relationship shown in $E q .5, Q=182 \mathrm{~mW} / \mathrm{cm}^{2}$. Over the whole area of the plotting medium $\left(2.83 \mathrm{~cm}^{2}\right)$ the energy exiting the plotting medium is $5.15 \mathrm{~W}$. The Peltier device is rated at $6 \mathrm{~W}$. The Parnon's cooling system operates at $86 \%$ efficiency. During a print, the NGM is being extruded into plotting medium that is $\sim 13^{\circ} \mathrm{C}$ less than the fastest solidification temperature. A dynamic heat 
1338 transfer model of NGM cooling in the plotting medium was not developed. NGM is 1339 extruded at $\sim 65^{\circ} \mathrm{C}$, however, $\sim 29^{\circ} \mathrm{C}$ above the fastest solidification temperature.

1340 Assuming that the thermal conductivities of NGM and the plotting medium are equal,

1341 then NGM would solidify the fastest if the plotting medium was also $\sim 29^{\circ} \mathrm{C}$ less than the 1342 fastest solidification temperature. There is another factor to consider, however, FDM 1343 relies on the previous layer remaining liquidous until the next layer is able to fuse. If the 1344 NGM solidifies too quickly the layers will not fuse together resulting in failed prints. 1345 While not quantified, we speculate that the limited ability of the Parnon's substrate 1346 cooling system is beneficial to the overall printing capability.

\section{Appendix}

List of Parnon major parts and items used for customization

- DOBOT MOOZ-2 machine, Shenzhen, China

- 12V DC Power Supply, 5V Power Supply

- Arduino SD Cardboard, Arduino UNO

- Heating Cartridge: Insertion Heater with Internal Temperature Sensor, for 3/8" Hole, 120V AC, 3" Long Heating Element, 200W, McMaster-Carr, USA

- Heating Controller: Programmable Temperature Controller, for Type K Thermocouple, McMaster-Carr, USA

- Limit Switch: Monoprice Maker Select 3D Printer 13860 Endstop Limit Switch, Amazon, USA

- Linear Actuator: NEMA-8 captive, HeydonKerk, USA

- Microstep Driver: TB6600 4A 9-42V Stepper Motor Driver CNC Controller, Amazon, USA

- Motor Driver: EasyDriver V4.5, SparkFun, USA

- Peltier Devices: Northbear Thermoelectric Cooler Peltier Refrigeration Cooling 


\section{Details on the Parnon customized print head}

1370 The customized print head includes the following parts (Fig. 4C): i) Bipolar stepper

1371 motor of the linear actuator, to provide the actuating force with a 40V input, controlled

1372 with Arduino. ii) 3D-printed custom connector (FormLabs Form2 3D printer, clear resin

1373 v4), used to house the stroke arm (iii) and to connect the motor (i) to the heat sink (v).

1374 iii) Stroke arm of the linear actuator with a 1.5" total actuation range and a 1:1 stroke of

$13753 \mu \mathrm{m}$. iv) Heating element, 3 " length, $3 / 8$ " diameter, $200 \mathrm{~W}$, set to $65^{\circ} \mathrm{C}$, used to provide

1376 the temperature required to keep the NGM in a liquid state. v) Custom heat sink

1377 designed to transfer heat from the heating element (iv) to the syringe (viii). vi)

1378 Connector (FormLabs Form2 3D printer, tough resin v5), used to connect the stroke

1379 arm (iii) to the plunger (vii). vii) Glass syringe plunger which has the custom connector

1380 (vi) adhered on the inside. viii) Glass syringe that houses the liquid NGM. ix) Metal luer-

1381 lock nozzle. $\mathbf{x}$ ) Copper wire heat induction system the prevents NGM solidification in the 1382 nozzle (ix). 